\title{
Exploring Noiseless Subsystems via Nuclear Magnetic Resonance
}

\author{
Evan M. Fortunato, ${ }^{1}$ Lorenza Viola,, , Marco A. Pravia, ${ }^{1}$ Emanuel Knill, ${ }^{2}$ \\ Raymond Laflamme, ${ }^{3}$ Timothy F. Havel, ${ }^{1}$ and David G. Cory ${ }^{1}$ \\ ${ }^{1}$ Department of Nuclear Engineering, Massachusetts Institute of Technology, Cambridge, MA 02139 \\ ${ }^{2}$ Los Alamos National Laboratory, Mail Stop B256, Los Alamos, NM 87545 \\ ${ }^{3}$ Department of Physics, University of Waterloo, Waterloo, ON Canada, N2L 3G1, and \\ Perimeter Institute for Theoretical Physics, 35 King Street N., Waterloo, ON Canada, N2J 2 W9
}

\begin{abstract}
Noiseless subsystems offer a general and efficient method for protecting quantum information in the presence of noise that has symmetry properties. A paradigmatic class of error models displaying non-trivial symmetries emerges under collective noise behavior, which implies a permutationally-invariant interaction between the system and the environment. We describe experiments demonstrating the preservation of a bit of quantum information encoded in a three qubit noiseless subsystem for general collective noise. A complete set of input states is used to determine the super-operator for the implemented one-qubit process and to confirm that the fidelity of entanglement is improved for a large, non-commutative set of engineered errors. To date, this is the largest set of error operators that has been successfully corrected for by any quantum code.

PACS numbers: 03.67.-a, 03.65.Yz, 76.60.-k, 89.70.+c
\end{abstract}

\section{INTRODUCTION}

Quantum information processing (QIP) holds the promise of solving problems in quantum simulation, quantum computation, and secure communication that have no known efficient solution in classical information processing [1]. While QIP can be, in principle, abstractly characterized without reference to the details of a specific implementation, physically realizing quantum information and its manipulation is essential to practically exploit its unique capabilities [2]. Real physical systems are invariably exposed to environmental noise and decoherence due to incomplete isolation from their surroundings, as well as to operational errors caused by imperfect manipulations. Thus, physical realizations of QIP are confronted with the challenge of achieving noise control during storage and processing of quantum information.

Thanks to a series of recent investigations, major progress has been witnessed on the theory front of reliable QIP. On one side, powerful "accuracy threshold theorems" for fault-tolerant quantum error correction (QEC) [3, 4, 5, 6, 7] ensure that, if the integrated effect of the noise per qubit and computational step remains sufficiently small, arbitrarily accurate QIP is still possible, in principle, with reasonable resource overheads. On the other side, alternative noise control techniques have become available as options complementing and expanding the applicability of conventional QEC. These methods include both passive error control codes based on decoherence-free subspaces (DFSs) [8, 9, 10] and noiseless subsystems (NSs) [11, 12, 13, 14], and active error suppression schemes relying on dynamical decoupling [15, 16, 17, 18, 19, 20], along with a variety of device-dependent schemes for reducing systematic and calibration errors [21, 22, 23, 24].

In retrospect, what constitutes the unifying conceptual feature of these advances, and what ultimately made them possible, is the realization that protecting quantum information against noise need not require the overall state of the physical device supporting QIP to be perfect [25]. Defining in what sense quantum information can be accurately stored in the noisy state of a physical system leads to think of all possible noise control options in terms of the emergence of logical subsystems (or "abstract" quantum particles) which are or can be made immune to noise [11, 12, 26] . In the former case, the occurrence of a NS directly ties into the existence of symmetry properties of the natural noise process, and error-free information storage is ensured without requiring active intervention [2, 11]. In the latter case, an external control action is instead necessary to appropriately symmetrize the dynamics [12, 14, 27] or to enforce noiselessness by a process that incorporates active recovery operations as well [11]. The basic intuition remains, nevertheless, unchanged.

While the implications of the subsystem approach are still being investigated, the idea of separating the abstract informationcarrying degrees of freedom from the implemented qubit degrees of freedom has proven useful beyond the original motivation of gaining noise protection. In particular, the notion of a subsystem has led to an operational prescription for realizing logical qubits in physical systems [2], and the analysis of the resources required to universally control subsystem-encoded qubits has resulted in an encoded universality approach [12, 28], where the notion of universality may be tailored to the set of physically available interactions. More recently, the idea of a NS has shown to also underlie topological approaches to QIP [29].

\footnotetext{
${ }^{*}$ Corresponding author: viola@lanl.gov
} 
From the experimental point of view, the significance of the NS notion has only recently begun to be explored. In particular, the first implementation of a non-trivial NS for general collective noise in a three-qubit liquid-state nuclear magnetic resonance (NMR) quantum information processor was reported by Viola et al. [30]. Here, we deepen our earlier investigation in two ways: by presenting an expanded description of the theoretical analysis and experimental methodology underlying [30]; by reporting additional experimental results which may further shed light on the relevance of NSs within present-day quantum information technology.

\section{THEORETICAL BACKGROUND}

\section{A. Noiseless subsystems: From simple examples to the general definition}

We begin by trying to build the intuition underlying the NS idea based on simple considerations and prototype examples [2, 26]. It is a well-known lesson in physics that the occurrence of symmetries in a system generally implies the existence of "conserved quantities", and that these can be exploited to ease the understanding of the system's behavior. In its essence, the NS approach adapts this lesson to the QIP-motivated task of achieving protection against noise. This is done by noticing that even though the system as a whole may be experiencing errors, some of its properties may still remain unaffected by them. Thus, if information can be represented in terms of the abstract degrees of freedom (DOFs) corresponding to such conserved quantities, noiselessness may be ensured in spite of the fact that the errors evolve the overall system's state.

This intuition applies to both classical and quantum information storage. A simple classical example arises by considering two bits subject to errors which either flip both bits with probability $p$, or leave them alone with probability $1-p$. This error model has the property that it preserves the parity $P(s)$ of a state $s$ of the bits, where $P(s)$ is defined as the sum (mod 2) of the bit string $s$. Thus, the two values of $P$ can be used together with the (non-conserved) value of, say, the first physical bit to label the four possible states of the two bits:

$$
\begin{aligned}
& 00 \leftrightarrow 0 \cdot 0 \\
& 01 \leftrightarrow 1 \cdot 0 \\
& 10 \leftrightarrow 1 \cdot 1 \\
& 11 \leftrightarrow 0 \cdot 1 .
\end{aligned}
$$

The above table establishes a correspondence between the state space of the physical system (left hand side) and the one of a pair of abstract subsystems (right hand side). In the resulting representation, the first member of the abstract pair (the parity bit) carries the information to be protected, while the second (the "syndrome" bit) experiences the effect of the errors. In this case, parity provides a classical NS, and the information resides in this protected DOF.

Many of the above features carry over to the quantum-mechanical case. In quantum systems, the presence of symmetries (hence of conserved quantities) is associated with the existence of operators that commute with all possible errors. First, consider a simple two-qubit example which corresponds to complete depolarization on qubit 2 . This error model is defined by the set of error operators $\left\{E_{a}\right\}=\left\{.5 \sigma_{a}^{(2)}, a=0, x, y, z\right\}, \sigma_{0}^{(2)}=\mathbb{1}^{(2)}$. Clearly, no joint state of the two qubits is protected. However, a one-qubit state can be stored in the first physical qubit without being affected by the errors: qubit 1 is a "trivial" quantum NS. Mathematically, this intuition is made precise by observing that, because $\left[E_{a}, \sigma_{u}^{(1)}\right]=0$ for every error and $u=x, y, z$, all the expectations of $\sigma_{u}^{(1)}$ - hence the state of qubit 1 - are protected from noise: $\sigma_{u}^{(1)}, u=x, y, z$, define the observables of this trivial NS qubit.

In general, knowing the symmetries of the error model suffices for identifying possible protected DOFs, in a way similar to what happens in the above simple example: one first determines the set of operators that commute with the possible errors, and then in this set identifies appropriate combinations that algebraically behave like the observables (the Pauli operators) for abstract qubits [2]. If $S$ is a (finite-dimensional) quantum system interacting with some environment $E$, a description of noise on $S$ which suffices for discussing error protection and error correction properties can be obtained by constructing the appropriate interaction algebra $\mathcal{A}$ [11]. Let the open-system evolution of $S$ involve coupling operators $J_{a}$, where the $J_{a}$ are traceless and we assume, for the moment, that the internal Hamiltonian $H_{S}$ of $S$ alone can be either set to zero or identified with one of the $J_{a}$. Then $\mathcal{A}$ contains all the complex linear combinations of arbitrary products of the $J_{a}$ and the identity. If $S$ is initialized in the state $\varrho_{i n}$, and the evolution is depicted in terms of a quantum operation [31],

$$
\varrho_{\text {in }} \mapsto \varrho_{\text {out }}=\mathcal{E}\left(\varrho_{\text {in }}\right)=\sum_{a} E_{a} \varrho_{\text {in }} E_{a}^{\dagger}, \quad \sum_{a} E_{a}^{\dagger} E_{a}=\mathbb{1},
$$

then $\mathcal{A}$ collects all the possible errors that the coupling to $E$ can induce for arbitrary strength or interaction time. The commutant $\mathcal{A}^{\prime}$ of $\mathcal{A}$, which collects all operators on $S$ that commute with arbitrary errors in $\mathcal{A}$, is the relevant structure to be used in generalizing the symmetry argument given above. By construction, both $\mathcal{A}$ and $\mathcal{A}^{\prime}$ are (multiplicative) sub-algebras of the full 
algebra $\mathcal{A}_{S}$ of operators on $S$. Within the Hamiltonian framework for the composite system $S, E$ adopted here, they are also naturally closed under the $\dagger$ operation, making some standard results from the representation theory of operator algebras directly applicable [32, 33, 34]. The symmetry properties of error models corresponding to $\mathcal{A}$ can be visualized by thinking of the largest group of unitary operators contained in $\mathcal{A}^{\prime}$ as the symmetry group for the problem.

Suppose that $S$ consists of $n$ physical qubits, in which case the state space $\mathcal{H} \simeq\left(\mathbb{C}^{2}\right)^{\otimes n}$, and $\mathcal{A}_{S}$ can be identified with the algebra of complex matrices $\operatorname{Mat}_{N}(\mathbb{C})$ acting on $\mathbb{C}^{N}, N=2^{n}$. Then three possibilities are worth examining:

- If $\mathcal{A}^{\prime}$ consists only of scalar multiples of the $\mathbb{1}$, no useful symmetries are present. Mathematically, this condition is equivalent to the fact that the error algebra $\mathcal{A}$ acts "irreducibly" on $\mathcal{H}$, hence $\mathcal{A}=\mathcal{A}_{S}$ by the Schur lemma [11, 32]. Because the error process is contributed by all operators on $S$, no protected DOF exists.

- Suppose instead that $\mathcal{A}^{\prime}$ is non-trivial, which implies that $\mathcal{A}$ is a proper sub-algebra of $\mathcal{A}_{S}=\operatorname{Mat}_{N}(\mathbb{C})$. If $\mathcal{A} \simeq \operatorname{Mat}_{m}(\mathbb{C})$ for some integer $m$, then one can show [32] that there exists a change of basis $U$ on $\mathcal{H}$, and integers $d, r$ with $m d+r=N$,

$$
U: \mathbb{C}^{N} \rightarrow \mathbb{C}^{m} \otimes \mathbb{C}^{d} \oplus \mathbb{C}^{r}
$$

such that, in the resulting representation, all error operators leave the factor $\mathbb{C}^{m}$ unaffected:

$$
U \mathcal{A} U^{\dagger}=\mathbb{1}_{m} \otimes \operatorname{Mat}_{d}(\mathbb{C}) \oplus \mathbb{O}_{r},
$$

where $\mathbb{O}_{r}$ accounts for the action on the remaining summand $\mathbb{C}^{r}$. Situations where a NS can be directly identified with a subset of the physical qubits belong to this category. The above trivial two-qubit example, for instance, corresponds to $N=4, m=d=2, r=0$.

- If $\mathcal{A}$ is again strictly contained in $\mathcal{A}_{S}$, but it cannot be identified with the operator algebra of a $m$-dimensional quantum DOF, a theorem from the representation theory of operator algebras (the Wedderburn theorem, [33], 34]; see also [35]) still implies that a transformation $U$ to a new basis exists where, as above, the action of the errors takes a simple blockfactorized form:

$$
U: \mathbb{C}^{N} \rightarrow \mathbb{C}^{m_{1}} \otimes \mathbb{C}^{d_{1}} \oplus \ldots \oplus \mathbb{C}^{m_{\ell}} \otimes \mathbb{C}^{d_{\ell}}
$$

with $\sum_{j} m_{j} d_{j}=N$, and

$$
U \mathcal{A} U^{\dagger}=\mathbb{1}_{m_{1}} \otimes \operatorname{Mat}_{d_{1}}(\mathbb{C}) \oplus \ldots \oplus \mathbb{1}_{m_{\ell}} \otimes \operatorname{Mat}_{d_{\ell}} .
$$

Because, in this representation, the action of any error operator in $\mathcal{A}$ is only experienced by the "syndrome factors" $\mathbb{C}^{d_{j}}$ in the decomposition (4), each of the co-factors $\mathbb{C}^{m_{j}}$ can be identified with the state space of a $m_{j}$-dimensional NS under $\mathcal{A}$ [11, 36.

It is worth noting that, with respect to the same basis ( 4 , the action of operators commuting with the errors becomes "dual" to the one of the errors themselves,

$$
U \mathcal{A}^{\prime} U^{\dagger}=\operatorname{Mat}_{m_{1}}(\mathbb{C}) \otimes \mathbb{1}_{d_{1}} \oplus \ldots \oplus \operatorname{Mat}_{m_{\ell}}(\mathbb{C}) \otimes \mathbb{1}_{d_{\ell}},
$$

implying that a non-trivial transformation is now enforced on the noiseless DOFs. Also, whenever $d_{j}=1$ for some $j$, the syndrome subsystem becomes effectively a classical DOF with a one-point configuration space. Thus, $\mathbb{C}^{m_{j}} \otimes \mathbb{C} \simeq \mathbb{C}^{m_{j}}$, and the $j$ th summand in the decomposition (4) can accordingly be identified with a DFS under $\mathcal{A}$ [11, 14].

Because of the abstract algebraic nature of the subsystem identification given by ( $(4)$ - (5), the mapping between the states of the information-carrying NSs and the states of the underlying physical qubits may in general become very indirect. However, the method for constructing a NS from appropriate observables in $\mathcal{A}^{\prime}$ may be applied in general [26]. We now make these considerations explicit in the situation that is relevant to the experimental implementation.

\section{B. Collective noise for three qubits}

The collective error behavior provides the paradigmatic situation for discussing passive noise control through both DFSs and NSs, and in particular for realizing the simplest non-trivial noiseless quantum subsystem. Collective error models have been extensively analyzed in the theory literature [8, 10, 11, 27, 37, and experimentally investigated in the QIP context in optical [38], trapped-ion [39], and liquid-state NMR [30, 40] devices. For a system $S$ composed of $n$ qubits as above, collective noise behavior arises whenever a single environment $E$ couples to the individual particles without distinguishing among them. This results in error models that are characterized by permutation symmetry. Whether or not the natural dynamics of $S$ alone, ruled 
by $H_{S}$, actually respects this symmetry (as assumed so far) is an important issue with both conceptual and practical implications. While deferring a more detailed discussion of this point to a later stage, we begin by examining the consequences of permutation symmetry.

In particular, let us focus on the situation where $S$ is composed of three qubits, implying that $\mathcal{H} \simeq \mathbb{C}^{8}$, and the full operator algebra $\mathcal{A}_{S} \simeq \operatorname{Mat}_{8}(\mathbb{C})$. For the purpose of characterizing collective error models, the crucial property is that only global error generators $J_{u}=\left(\sigma_{u}^{(1)}+\sigma_{u}^{(2)}+\sigma_{u}^{(3)}\right) / 2, u=x, y, z$, may be present in the system-environment interaction. By definition, $J_{u}$ is the projection of the total spin angular momentum along the $\hat{u}$-axis (in units $\hbar$ ). In NMR with spin-1/2 nuclei, for instance, interactions of this type may arise from uniform, fully-correlated magnetic fields which fluctuate in direction and strength, leading to evolutions which can be semi-classically described as [41]

$$
|\Psi\rangle_{123} \mapsto\left|\Psi^{\prime}\right\rangle_{123}=e^{-i\left(\theta_{x} J_{x}+\theta_{y} J_{y}+\theta_{z} J_{z}\right)}|\Psi\rangle_{123},
$$

for random variables $\theta_{u}, u=x, y, z$. In the formalism of quantum operations, collective error processes are characterized by completely positive dynamical maps of the form (1), where the possible errors $E_{a}$ are constrained to commute with all possible particle permutations. Thus, the largest interaction algebra $\mathcal{A}_{c}$ resulting from arbitrary collective interactions consists of all the totally symmetric operators on three qubits. Because the dimension of the subspace of totally symmetric operators for $n$ qubits is given by $(n+1)(n+2)(n+3) / 6$ [42], $\mathcal{A}_{c}$ is a 20-dimensional sub-algebra of $\mathcal{A}_{S}$. This implies that a description of the most general collective error model on three qubits can be accomplished by using an error basis with at most 20 (linearly independent) operators, out of the possible 64 needed for representing arbitrary noise in the absence of symmetries. By writing

$$
\mathcal{A}_{c}=\operatorname{span}\left\{\Sigma_{a} \mid a=0, \ldots, 19\right\} \subset \mathcal{A}_{S},
$$

an explicit basis of operators $\Sigma_{a}$ can be constructed by fully symmetrizing the standard Pauli product operator basis for three qubits. Let us introduce compact notations to describe operators that are invariant under the full set of qubit permutations:

$$
\begin{aligned}
\widehat{Z Z} & =\sigma_{z}^{(1)} \sigma_{z}^{(2)}+\sigma_{z}^{(2)} \sigma_{z}^{(3)}+\sigma_{z}^{(3)} \sigma_{z}^{(1)}, \\
\widehat{Z X} & =\sigma_{z}^{(1)} \sigma_{x}^{(2)}+\sigma_{x}^{(1)} \sigma_{z}^{(2)}+\sigma_{z}^{(1)} \sigma_{x}^{(3)}+\sigma_{x}^{(1)} \sigma_{z}^{(3)}+\sigma_{z}^{(2)} \sigma_{x}^{(3)}+\sigma_{x}^{(2)} \sigma_{z}^{(3)}, \\
\widehat{Z Z Z} & =\sigma_{z}^{(1)} \sigma_{z}^{(2)} \sigma_{z}^{(3)}, \\
\widehat{Z Z X} & =\sigma_{z}^{(1)} \sigma_{z}^{(2)} \sigma_{x}^{(3)}+\sigma_{x}^{(1)} \sigma_{z}^{(2)} \sigma_{z}^{(3)}+\sigma_{z}^{(1)} \sigma_{x}^{(2)} \sigma_{z}^{(3)}, \\
\widehat{X Y Z} & =\sigma_{x}^{(1)} \sigma_{y}^{(2)} \sigma_{z}^{(3)}+\sigma_{y}^{(1)} \sigma_{x}^{(2)} \sigma_{z}^{(3)}+\sigma_{z}^{(1)} \sigma_{y}^{(2)} \sigma_{x}^{(3)}+\sigma_{x}^{(1)} \sigma_{z}^{(2)} \sigma_{y}^{(3)}+\sigma_{y}^{(1)} \sigma_{z}^{(2)} \sigma_{x}^{(3)}+\sigma_{z}^{(1)} \sigma_{x}^{(2)} \sigma_{y}^{(3)},
\end{aligned}
$$

and so forth. Then a basis for $\mathcal{A}_{c}$ is given by the $\mathbb{1}$, the three linear operators $J_{u}$, the six quadratic operators $\widehat{X X}, \widehat{Y Y}$, $\widehat{Z Z}, \widehat{X Z}, \widehat{X Y}, \widehat{Y Z}$, and the ten cubic operators resulting from the above construction.

As noted earlier, the symmetry properties of a given error model appear explicitly in the commutant of the error algebra. In the case of $\mathcal{A}_{c}$, the commutant $\mathcal{A}_{c}^{\prime}$ contains the subgroup $\Pi$ of unitary operators that implement permutations of the particles e.g., a swap $\pi_{12}$ between qubit 1, 2 means $\pi_{12}|i\rangle_{1}|j\rangle_{2}|k\rangle_{3}=|i\rangle_{2}|j\rangle_{1}|k\rangle_{3}=|j\rangle_{1}|i\rangle_{2}|k\rangle_{3}$. In fact, one can show that the whole $\mathcal{A}_{c}^{\prime}$ consists of linear combinations of operators in $\Pi$, expressing the fact that $\mathcal{A}_{c}^{\prime}$ coincides with the group algebra $\mathbb{C S}_{3}$ of the permutation group $\mathcal{S}_{3}$ under the above representation in $\mathcal{H}[14,26,32]$. Physically, because the error generators $J_{u}$ are also the generators for the global rotations of the qubits, $\mathcal{A}_{c}^{\prime}$ can be regarded as containing the operators which remain invariant under such rotations. Thus, operators in $\mathcal{A}_{c}^{\prime}$ can be constructed from the identity and the simplest invariant operators [2, 11]:

$$
s_{12}=\vec{\sigma}^{(1)} \cdot \vec{\sigma}^{(2)}, \quad s_{23}=\vec{\sigma}^{(2)} \cdot \vec{\sigma}^{(3)}, \quad s_{31}=\vec{\sigma}^{(3)} \cdot \vec{\sigma}^{(1)},
$$

where - denotes the usual dot product. Note that $s_{j k}$ is nothing but the Heisenberg spin coupling between spins $j, k$. As it turns out, every operator in $\mathcal{A}_{c}^{\prime}$ can be realized, in principle, through the application of Heisenberg Hamiltonians of the form (10] [43]. By observing that the total angular momentum observable $J^{2}=\vec{J} \cdot \vec{J}$ simply rewrites in terms of the above operators, collective symmetry immediately implies a conserved quantity, given by the eigenvalues $j(j+1)$ of $J^{2}$.

\section{Abelian error models}

Suppose that the system-environment interaction is contributed by a single global error generator $J_{\hat{v}}=\vec{J} \cdot \hat{v}, \hat{v} \cdot \hat{v}=1$. Then, in the absence of an independent quantizing direction (provided, for instance by $H_{S}$ ), the resulting interaction algebra is abelian, and the corresponding error models accounts for collective decoherence (unrecoverable loss of phase information) with respect to the fixed basis of eigenstates of $\sigma_{\hat{v}}$. The choice $\hat{v}=\hat{z}$ singles out the $z$ basis. For three qubits, the relevant error algebra $\mathcal{A}_{z}$ can be constructed from the generator $J_{z}$ and the $\mathbb{1}$, and can be identified with the sub-algebra of $\mathcal{A}_{c}$ spanned by the four axially symmetric and permutation-invariant operators, i.e.

$$
\mathcal{A}_{z}=\operatorname{span}\left\{\mathbb{1}, J_{z}, \widehat{Z Z}, \widehat{Z Z Z}\right\} \subset \mathcal{A}_{c},
$$


For instance, an error model in this class that will be of practical significance is a full-strength (or "crusher", borrowing from the NMR terminology) collective $z$-dephasing on three qubits, which may be described by a quantum operation $\mathcal{E}_{z}$ with Kraus operation elements $K_{a}^{z}, a=0, \ldots, 3$ :

$$
\begin{aligned}
K_{0}^{z} & =\frac{1}{8}\left(\mathbb{1}+2 J_{z}+\widehat{Z Z}+\widehat{Z Z Z}\right), \\
K_{1}^{z} & =\frac{1}{8}\left(\mathbb{1}-2 J_{z}+\widehat{Z Z}-\widehat{Z Z Z}\right), \\
K_{2}^{z} & =\frac{1}{8}\left(3 \mathbb{1}+2 J_{z}-\widehat{Z Z}-3 \widehat{Z Z Z}\right), \\
K_{3}^{z} & =\frac{1}{8}\left(3 \mathbb{1}-2 J_{z}-\widehat{Z Z}+3 \widehat{Z Z Z}\right) .
\end{aligned}
$$

In a similar way, collective decoherence about the $\hat{x}$ axis can be described by effectively switching from the $z$ to the $x$ basis, i.e. by mapping $\mathcal{A}_{z} \mapsto \mathcal{A}_{x}=H \mathcal{A}_{z} H$, where $H$ represents a collective Hadamard transform. Accordingly, a crusher collective $x$-error process corresponds to $\mathcal{E}_{x}=\left\{K_{a}^{x}\right\}$, with operation elements obtained from (12) via the appropriate rotations.

For later purposes of comparison between encoded and un-encoded information, the description of the noise process induced by a three-qubit error model on a physical information-carrying qubit will be useful. In general, by treating one of the qubits as the data qubit $(d)$ and the remaining ones as ancillae $\left(a_{1}, a_{2}\right)$, the partial trace operation over the ancillae

$$
\rho_{\text {in }}=\operatorname{Tr}_{\mathrm{a}_{1}, \mathrm{a}_{2}}\left\{\varrho_{i n}\right\} \mapsto \operatorname{Tr}_{\mathrm{a}_{1}, \mathrm{a}_{2}}\left\{\mathcal{E}\left(\varrho_{i n}\right)\right\}=\mathcal{Q}\left(\rho_{\text {in }}\right)
$$

associates to a three-qubit process $\mathcal{E}$ a one-qubit process $\mathcal{Q}$ on the data qubit d alone, provided that the latter is initially uncorrelated with the ancillae and the initial state $\left|a_{1} a_{2}\right\rangle$ is known. For the experimental realization, the choice $\left|a_{1} a_{2}\right\rangle=|00\rangle$ will be relevant. It is then readily seen that the above $\mathcal{E}_{z}$ process corresponds, for instance, to applying a one-qubit map of the form

$$
\rho \mapsto E_{+}^{z} \rho E_{+}^{z}+E_{-}^{z} \rho E_{-}^{z},
$$

where $E_{ \pm}^{z}=\left(\mathbb{1} \pm \sigma_{z}\right) / 2$ are the usual $z$-idempotents, $\left(E_{ \pm}^{z}\right)^{2}=E_{ \pm}^{z}$. As expected from physical intuition, this process is nothing but crusher phase damping on the (un-encoded) data qubit.

\section{Non-abelian error models}

Whenever two non-commuting error generators are relevant, the interaction algebra describing the resulting error process is non-abelian. In practice, we shall be interested at error models obtainable by using abelian noise processes as building blocks. Let $\mathcal{E}_{x}, \mathcal{E}_{y}, \mathcal{E}_{z}$ denote crusher dephasing about $\hat{x}, \hat{y}, \hat{z}$, respectively, with associated error algebras $\mathcal{A}_{x}, \mathcal{A}_{y}, \mathcal{A}_{z}$, as above. A simple way for inducing non-abelian error processes is through the sequential composition of abelian errors along different axes. In particular, crusher isotropic collective decoherence corresponds to cascading crusher noise processes about all three axes [44]. For instance, $\mathcal{E}_{y} \mathcal{E}_{z} \mathcal{E}_{x}=\mathcal{E}_{y z x}$, with Kraus operators specified by the 64 (linearly dependent) products $K_{c}^{y} K_{b}^{z} K_{a}^{x}, a, b, c=0, \ldots, 3$, of the operators given in the previous subsection. While composite noise processes of this sort may not naturally occur in physical systems, they can be enforced in liquid-state NMR using readily available non-unitary control methods to be described later (see also [44]). In terms of error algebras, the operators describing the composite noise process $\mathcal{E}_{y z x}$ can be thought to belong to an error algebra $\mathcal{A}_{y z x}$ which arises from the multiplication of the single-axis algebras,

$$
\mathcal{A}_{y z x}=\mathcal{A}_{y} \mathcal{A}_{z} \mathcal{A}_{x}=\operatorname{span}\left\{K_{c}^{y} K_{b}^{z} K_{a}^{x}, K_{a}^{x} K_{b}^{z} K_{c}^{y}\right\} \subseteq \mathcal{A}_{c} .
$$

In fact, one can show that $\mathcal{A}_{y z x}$ is the full collective algebra $\mathcal{A}_{c}$ by checking that a basis $\Sigma_{a}$ of permutation-invariant operators (such as the one given in (9)) is contained in $\mathcal{A}_{y z x}$. It also turns out that the full $\mathcal{A}_{c}$ may be generated from the composition of abelian error processes involving two non-commuting axes, e.g. $\mathcal{E}_{z} \mathcal{E}_{x}=\mathcal{E}_{z x}$.

By reasoning as in the abelian case, the one-qubit map $\mathcal{Q}$ describing the effect of a crusher composite noise processes at the single-qubit level can be derived by evaluating the partial trace (13) on the appropriate sets of 16 (for two-axes noise) or 64 (for three-axes noise) Kraus operators. The result is effective full depolarization on the physical data qubit, corresponding to a map of the form

$$
\rho \mapsto \frac{1}{4}\left(\rho+\sum_{u=x, y, z} \sigma_{u} \rho \sigma_{u}\right)
$$




\section{E. The role of the self-Hamiltonian}

From a physical point of view, it is worth pointing out that while noise processes that involve both quantum decoherence (phase damping) and dissipation (amplitude damping) always correspond to non-abelian error algebras, a non-commutative error algebra does not necessarily indicate the presence of genuine energy dissipation in the system. In general, in the absence of an internal Hamiltonian, $H_{S}=0$, every noise process is effectively equivalent to (adiabatic) decoherence. For instance, because all the basic operation elements in (12) are Hermitian, crusher collective dephasing as defined above is, consistently, a unital process, describing damping of phase information in the $z$ basis. Composition of dephasing operations along non-commuting axes results in depolarization, which is still unital and can be regarded as decoherence in all bases.

Because $H_{S}$ is rarely zero in real systems, the interplay between the internal dynamics and the actual error generators $J_{a}$ turns out to be crucial for characterizing the overall open-system dynamics. Clearly, it is always possible to construct the interaction algebra $\mathcal{A}$ by including $H_{S}$, as done so far, among the defining interaction operators. In particular, the assumption $H_{S}=\epsilon J_{z}$ is implicit in the original definition of the collective noise model [8]. Regardless of whether $H_{S}$ commutes or not with the $J_{a}$, if a non-trivial NS is supported by the algebra $\mathcal{A}$ constructed in this way, this NS is completely stable against time evolution. Therefore this procedure is, in principle, ideal for devising robust quantum memories.

However, including $H_{S}$ among the defining operators for $\mathcal{A}$ is not desirable when the $\mathcal{A}$ becomes irreducible (thus without useful symmetries) or when $H_{S}$ is regarded as a resource for effecting noise-protected manipulations of information. Suppose that an error algebra $\tilde{\mathcal{A}}$ is defined starting from the $\mathbb{1}$ and the $J_{a}$ alone. Then $H_{S}$ may or may not belong to $\tilde{\mathcal{A}}^{\prime}$. From the point of view of reliable QIP, situations falling into the first category are the most favorable, as the natural Hamiltonian directly implements a non-trivial logical evolution on any NS supported by $\tilde{\mathcal{A}}\left[19,40,45\right.$. If $H_{S} \notin \tilde{\mathcal{A}}^{\prime}$ instead, then $H_{S}$ may still preserve a given NS [37, 40], but most likely it will have the undesired effect of causing leakage outside the intended space. While various schemes are available in principle to cope with these effects [1, 46], it is not clear to what extent these may be viable with realistic control resources.

In liquid-state NMR systems, collective error models may naturally play a role in describing relaxation from fully correlated fields in homo-nuclear species [41, 47]. However, the assumption that $H_{S}$ is proportional to $J_{z}$ is invalid due to the chemical shift effects. For the specific molecule used in the current implementation, $H_{S}$ (to be explicitly given later) will turn out to satisfy none of the properties of belonging to the full collective error algebra $\mathcal{A}_{c}$ or its commutant $\mathcal{A}_{c}^{\prime}$. Nevertheless, the system may be used to demonstrate how robustness against $\mathcal{A}_{c}$ can be achieved by constructing an appropriate NS.

\section{F. The three spin-1/2 noiseless subsystem}

Because, as noted earlier, the eigenvalue $j$ of the total angular momentum $J^{2}$ is conserved, simultaneous eigenstates of $J^{2}, J_{z}$ are a natural basis to describe the state of the three particles. The possible values of $j$ are $j=3 / 2,1 / 2$, corresponding to a decomposition of $\mathcal{H}$ as the direct sum of two invariant subspaces, $\mathcal{H} \simeq \mathcal{H}_{3 / 2} \oplus \mathcal{H}_{1 / 2}$, respectively. The quantum numbers $j, j_{z}$ suffice for completely labeling basis states in $\mathcal{H}_{3 / 2}$, which is the four-dimensional subspace spanned by totally symmetric states (i.e., states in $\mathcal{H}_{3 / 2}$ transform under particle permutations as the one-dimensional symmetric irreducible representation of $\mathcal{S}_{3}$ ). However, with $j=1 / 2$ and $j_{z}= \pm 1 / 2$ this is no longer true for the subspace $\mathcal{H}_{1 / 2}$, which is also four-dimensional as the eigenvalue $j=1 / 2$ is in fact doubly degenerate. Physically, this degeneracy accounts for the fact that there are two distinct paths for obtaining a total angular momentum $j=1 / 2$ out of three elementary $1 / 2$ spins. Let $\ell=0,1$ be an additional quantum number that labels these two possible paths. Because collective errors do not have access to the quantum numbers of the individual spins, and the resulting global quantum numbers are the same in both paths, the noise can neither distinguish which value of $\ell$ is realized, nor change that value. Thus, $\ell$ corresponds to a conserved, two-dimensional DOF under the noise. In fact, this is the NS we are seeking [2, 11, 48].

More formally, basis states in $\mathcal{H}_{1 / 2}$ are labeled by two quantum numbers, $\left|\ell, j_{z}\right\rangle$, with $\ell=0,1, j_{z}= \pm 1 / 2$. For fixed $\ell$ (fixed path), the resulting subspace carries a copy of the two-dimensional irreducible representation of the angular momentum group $s u(2)$ corresponding to $j=1 / 2$. There are two such copies, and operators in $\mathcal{A}_{c}$ do not mix them and act identically on both. For fixed $j_{z}$, one obtains instead a copy of the two-dimensional irreducible representation [2 1] (in Young tableau notation) of the permutation group $\mathcal{S}_{3}$ [32, 49]. There are again two such copies, and now operators in $\mathcal{A}_{c}^{\prime}$ do not mix them and act identically on both [50]. With respect to the interaction algebra $\mathcal{A}_{c}$, we can thus identify the DOF corresponding to $\ell$ with a subsystem $L$, which is fully protected against errors, and the DOF corresponding to $j_{z}$ with a syndrome spin- $1 / 2$ subsystem $Z$, which instead experiences the errors. We write $\mathcal{H}_{1 / 2} \leftrightarrow \mathcal{H}_{L} \otimes \mathcal{H}_{Z}$, and make this identification explicit through the following correspondence with states in the computational basis [2]:

$$
\begin{aligned}
|0\rangle_{L} \otimes|+1 / 2\rangle_{Z} & \leftrightarrow \frac{1}{\sqrt{3}}\left(|001\rangle+\omega|010\rangle+\omega^{2}|100\rangle\right), \\
|0\rangle_{L} \otimes|-1 / 2\rangle_{Z} & \leftrightarrow \frac{1}{\sqrt{3}}\left(|110\rangle+\omega|101\rangle+\omega^{2}|011\rangle\right),
\end{aligned}
$$




$$
\begin{aligned}
|1\rangle_{L} \otimes|+1 / 2\rangle_{Z} & \leftrightarrow \frac{1}{\sqrt{3}}\left(|001\rangle+\omega^{2}|010\rangle+\omega|100\rangle\right), \\
|1\rangle_{L} \otimes|-1 / 2\rangle_{Z} & \leftrightarrow \frac{1}{\sqrt{3}}\left(|110\rangle+\omega^{2}|101\rangle+\omega|011\rangle\right),
\end{aligned}
$$

where $\omega=\exp (i 2 \pi / 3)$. Note that two of the totally symmetric states spanning $\mathcal{H}_{3 / 2}$ are $|000\rangle$, $|111\rangle$, while the remaining two are obtained from (17) by dropping the $\omega, \omega^{2}$ phase factors. Thus, the full set of basis states for $\mathcal{H}_{3 / 2}$ and $\mathcal{H}_{1 / 2}$ provides an explicit realization of the general state space decomposition given in (4), with $U$ being the rotation needed to bring the computational basis to the appropriate angular momentum basis, and $N=8, m_{1}=1, d_{1}=4, m_{2}=2, d_{2}=2$, respectively. Equivalently, the NS qubit living in $\mathcal{H}_{L}$ can be identified by combining the $\mathbb{1}$ and the invariant operators $(10)$ into three operators $\sigma_{u}^{(L)} \in \mathcal{A}_{c}^{\prime}$ that behave algebraically like the Pauli operators. This gives [2]

$$
\sigma_{x}^{(L)}=\frac{1}{2}\left(\mathbb{1}+s_{12}\right) P_{1 / 2}, \quad \sigma_{y}^{(L)}=\frac{\sqrt{3}}{6}\left(s_{23}-s_{31}\right) P_{1 / 2}, \quad \sigma_{z}^{(L)}=i \sigma_{x}^{(L)} \sigma_{y}^{(L)},
$$

where $P_{1 / 2}=\mathbb{1} / 2-\left(s_{12}+s_{23}+s_{31}\right) / 6$ denotes the projector onto the subspace $\mathcal{H}_{1 / 2}$. Note that the logical $\sigma_{x}^{(L)}$ observable is simply the restriction to $\mathcal{H}_{1 / 2}$ of the permutation $\pi_{12}$ swapping qubits 1 and 2 .

\section{G. Collective operators in the noiseless subsystem/syndrome representation}

It is instructive to take a closer look at the action of the error generators $J_{u}$ and of some relevant collective error processes directly in terms of the decomposition

$$
U \mathcal{H}=\mathcal{H}_{3 / 2} \oplus \mathcal{H}_{1 / 2} \leftrightarrow \mathcal{H}_{3 / 2} \oplus \mathcal{H}_{L} \otimes \mathcal{H}_{Z}
$$

where $U$ effects the change of basis mentioned above and the subsystem identification within $\mathcal{H}_{1 / 2}$ is explicitly given by $(17)$. By using the identity $1+\omega+\omega^{2}=0$, it is easy to verify that the restriction of the collective noise generators $J_{u}$ to the $\mathcal{H}_{1 / 2}$ subspace acts as follows:

$$
\begin{aligned}
& 2 J_{x} P_{1 / 2} \leftrightarrow \mathbb{1}^{(L)} \otimes\left(-\sigma_{x}^{(Z)}\right), \\
& 2 J_{y} P_{1 / 2} \leftrightarrow \mathbb{1}^{(L)} \otimes\left(-\sigma_{y}^{(Z)}\right), \\
& 2 J_{z} P_{1 / 2} \leftrightarrow \mathbb{1}^{(L)} \otimes\left(+\sigma_{z}^{(Z)}\right) .
\end{aligned}
$$

Thus, the $J_{u}$ act as single-qubit errors on the syndrome subsystem alone. Explicitly, this means that if, for instance, the initial state of the system is given by

$$
P_{1 / 2}|\Psi\rangle_{123}=|\Psi\rangle=(\alpha|0\rangle+\beta|1\rangle)_{L} \otimes(\gamma|+1 / 2\rangle+\delta|-1 / 2\rangle)_{Z},
$$

for appropriate coefficients, then the result of a collective rotation by, say, $\theta_{y}$ about $\hat{y}$ is

$$
\begin{aligned}
e^{-i \theta_{y} J_{y}}|\Psi\rangle & =(\alpha|0\rangle+\beta|1\rangle)_{L} \otimes e^{+i \theta_{y} \sigma_{y}^{(Z)} / 2}(\gamma|+1 / 2\rangle+\delta|-1 / 2\rangle)_{Z} \\
& =(\alpha|0\rangle+\beta|1\rangle)_{L} \otimes\left(\left(\gamma \cos \left(\theta_{y} / 2\right)+\delta \sin \left(\theta_{y} / 2\right)\right)|+1 / 2\rangle+\left(\delta \cos \left(\theta_{y} / 2\right)-\gamma \sin \left(\theta_{y} / 2\right)\right)|-1 / 2\rangle\right)_{Z},
\end{aligned}
$$

while a collective $z$ rotation is simply

$$
\begin{aligned}
e^{-i \theta_{z} J_{z}}|\Psi\rangle & =(\alpha|0\rangle+\beta|1\rangle)_{L} \otimes e^{-i \theta_{z} \sigma_{z}^{(Z)} / 2}(\gamma|+1 / 2\rangle+\delta|-1 / 2\rangle)_{Z} \\
& =(\alpha|0\rangle+\beta|1\rangle)_{L} \otimes\left(\gamma e^{-i \theta_{z} / 2}|+1 / 2\rangle+\delta e^{+i \theta_{z} / 2}|-1 / 2\rangle\right)_{Z}
\end{aligned}
$$

and so on. Two observations are worth making. First, if either $\delta$ or $\gamma$ is zero, then (23) is a direct manifestation of the fact that each of the pairs of states in (17) with fixed $j_{z}$ is a one-qubit DFS against pure $z$ noise [50]. As explained in Sect. IIIB, initialization of the ancillae qubits as $\left|\mathrm{a}_{1} \mathrm{a}_{2}\right\rangle=|00\rangle$ will correpond to encode into the $j_{z}=-1 / 2$ subspace, thus $\gamma=0$ in the implementation. Second, Eqs. (22) and (23) together show that encoding into the $Z$ subsystem would instead result in a qubit fully controllable in terms of homogeneous local unitaries [51] i.e., transformations of the form $U^{(1)} \otimes U^{(2)} \otimes U^{(3)}$ on the physical qubits - for instance, non-selective ("hard") $\pi$ pulses about two non-commuting axes. 
A procedure similar to the one outlined above may be applied to picture the effect of an arbitrary, known error model. Take, for instance, crusher collective $z$-dephasing with Kraus operators $\mathcal{E}_{z}=\left\{K_{a}^{z}\right\}$ given in (12). One finds that

$$
\begin{aligned}
& \widehat{Z Z} P_{1 / 2} \leftrightarrow \mathbb{1}^{(L)} \otimes\left(-\mathbb{1}^{(Z)}\right), \\
& \widehat{Z Z Z} P_{1 / 2} \leftrightarrow \mathbb{1}^{(L)} \otimes\left(-\sigma_{z}^{(Z)}\right) .
\end{aligned}
$$

Thus, when restricted to $\mathcal{H}_{1 / 2}$, the action of both $K_{0}^{z}$ and $K_{1}^{z}$ is zero, whereas

$$
\begin{aligned}
K_{2}^{z} P_{1 / 2} & \leftrightarrow \mathbb{1}^{(L)} \otimes E_{+}^{(Z)}, \\
K_{3}^{z} P_{1 / 2} & \leftrightarrow \mathbb{1}^{(L)} \otimes E_{-}^{(Z)} .
\end{aligned}
$$

This just means that the action of crusher collective phase errors on the physical system can be pictured as a crusher phase damping channel on the syndrome subsystem $Z$ alone. Because, as we shall also comment later, the state of the latter abstract subsystem is mapped, upon decoding, into the state of a physical ancilla qubit carrying the error syndrome [26], this means that the physical syndrome subsystem will have experienced full phase damping under the same conditions. By the same reasoning, the action of crusher composite noise processes can be understood in terms of the composition of phase-damping channels affecting the $Z$ subsystem along various axes, translating into depolarization of the physical syndrome subsystem. These observations will be corroborated by experiment.

\section{H. Verifying infinite-distance error-correcting properties}

As a result of the above analysis, quantum information encoded in the $L$ subsystem is protected indefinitely in time, without requiring any active intervention. In the language of QEC [11], this stability against the full collective algebra $\mathcal{A}_{c}$ characterizes $L$ as an infinite-distance QEC code for arbitrary collective errors. Formally, this follows from the fact that to the NS one can associate a QEC in the usual (subspace) sense by choosing an initial reference state $|\varepsilon\rangle_{Z}$ in the syndrome subsystem (corresponding to "no error"), and by letting the code subspace $\mathcal{C}$ be defined by

$$
\mathcal{C}=\operatorname{span}\left\{|0\rangle_{C}=|0\rangle_{L} \otimes|\varepsilon\rangle_{Z},|1\rangle_{C}=|1\rangle_{L} \otimes|\varepsilon\rangle_{Z}\right\} .
$$

Then the basis states of $\mathcal{C}$ verify the necessary and sufficient conditions for recovery from all errors in $\mathcal{A}_{c}$ [25], 52],

$$
\left\langle i_{C}\left|E_{a}^{\dagger} E_{b}\right| j_{C}\right\rangle=\alpha_{a b} \delta_{i, j}, \quad \forall E_{a}, E_{b} \in \mathcal{A}_{c},
$$

for appropriate coefficients $\alpha_{a b}$-independent of the logical index $i, j$. Note that, a priori, infinite-distance behavior as expressed by (27) applies regardless of whether $L$ is supported by a DFS or by a proper NS. For a DFS, the syndrome state is fixed thus it can be effectively disregarded. For a NS, however, the fact that errors are allowed to evolve the state $|\varepsilon\rangle_{Z}$ non-trivially implies that the latter may be effectively arbitrary. In both situations, no recovery is needed for maintaining information in $L[11]$.

In a NS-QEC experiment, one is interested at inferring robustness properties of the encoded information by looking at the code performance under a given set of quantum processes. Suppose that, as mentioned in the previous subsection, verification is constrained to having initialized the syndrome subsystem in the state $|-1 / 2\rangle_{Z}$. What kind of conclusions can one draw? The analysis is relatively simple under the assumption of perfect implementation fidelity. The relevant points can be summarized as follows:

- Verifying that quantum information is preserved under the implementation of an error process with Kraus operators $\left\{K_{a}\right\}$ implies stability under any error operator $K \in \operatorname{span}\left\{K_{a}\right\}[25]$.

- If the set of correctable error operators contains an error algebra $\mathcal{A}$ i.e., $\operatorname{span}\left\{K_{a}\right\} \supseteq \mathcal{A}$, the implementation verifies infinite-distance behavior under $\mathcal{A}[11]$.

- Verifying a NS under $\mathcal{A}$ requires verifying infinite-distance QEC for every possible initial state of the syndrome subsystem.

These observations can be applied to analyze both abelian and non-abelian collective error models. For instance, suppose we observe stability under crusher $x$ noise. Then we can conclude that operators in $\mathcal{A}_{x}$ have identity action on $L$ when $Z$ is initialized to $|-1 / 2\rangle_{Z}$. However, because the state $|+1 / 2\rangle_{Z}$ can be reached from $|-1 / 2\rangle_{Z}$ by application of error operators in $\mathcal{A}_{x}$, one has effectively verified a NS against $\mathcal{A}_{x}$. While a similar argument applies to $y$ noise, the state of the $Z$ subsystem is preserved under $z$ noise. Thus, observation of stability under $\mathcal{A}_{z}$ for fixed initialization in $|-1 / 2\rangle_{Z}$ only implies the verification of a DFS-behavior under $\mathcal{A}_{z}$. A proper NS-behavior under the full collective $\mathcal{A}_{c}$ can be inferred, in principle, in various ways. 
Keeping the experimental $|-1 / 2\rangle_{Z}$ preparation constraint in mind, the simplest procedure is to ensure stability under a family of quantum processes whose sets of Kraus operators globally span $\mathcal{A}_{c}$. For instance, one can check that

$$
\operatorname{span}\left\{K_{a}^{u} K_{b}^{v}, K_{b}^{v} K_{a}^{u} \mid a, b=0, \ldots, 3\right\}=\mathcal{A}_{c}
$$

for any choice of a composite crusher process which involves two non-commuting axes $\hat{u}, \hat{v}$ e.g., $\mathcal{E}_{z y}$ and $\mathcal{E}_{y z}$. The implemented set of processes are discussed in Sect. IVB-C. One may notice that the error operators describing $\mathcal{E}_{y z}$ are obtainable as a subset of the errors operators induced by $\mathcal{E}_{y z x}$. Thus, the implemented set is sufficient to infer that a NS for the most general collective noise has verified, at least in the limit of sufficiently high fidelity. Establishing what minimum fidelity threshold is required for inferring NS-verification under non-ideal conditions is a separate interesting issue, whose analysis is beyond the scope of this work.

The three-qubit NS turns out to provide the smallest code capable to correct one qubit against the full $\mathcal{A}_{c}$. The same, infinitedistance protection can be accomplished by using a DFS, but the most efficient DFS requires four physical qubits [8]. It is worth noting that, for a given physical system, more efficient codes may exist if additional symmetries are present beside the permutational one. If, for instance, axial symmetry also applies (i.e., the error model belongs to the class of collective single-axis phase damping), then a three-dimensional subspace of $\mathcal{H}$ may be protected with infinite distance by a DFS (corresponding to a fixed $j_{z}$ eigenvalue, $j_{z}= \pm 1 / 2$ ). The situation is summarized in Table I.

\section{EXPERIMENTAL DESIGN AND METHODS}

\section{A. Liquid-state nuclear magnetic resonance}

All experiments were performed using liquid-state NMR techniques on a sample of ${ }^{13} \mathrm{C}$ labeled alanine (Fig. 11) in $\mathrm{D}_{2} \mathrm{O}$ solution, using a $300 \mathrm{MHz}$ Bruker AVANCE spectrometer. The spin's evolution is governed by the internal Hamiltonian $H_{S}$, which in the weak coupling limit is accurately described by

$$
H_{S}=\pi \sum_{k=1,2,3} \nu_{k} \sigma_{z}^{k}+\frac{\pi}{2} \sum_{j>k=1}^{3} J_{k j} \sigma_{z}^{k} \sigma_{z}^{j},
$$

where $\nu_{k}$ represents the chemical shift frequency of the $k$ th spin, and $J_{k j}$ the coupling constant between spins $k$ and $j$, respectively (in $\mathrm{Hz}$ units). Radio frequency (RF) pulses are used to modulate the dynamics to produce the desired net evolution in the spin frame where the above internal Hamiltonian is defined. The interaction with the control field generated from a single transmitter has the form [24]

$$
H_{e x t}\left(\omega_{R F}, \phi, \omega, t\right)=\sum_{k} e^{-i\left(\omega_{R F} t+\phi\right) \sigma_{z}^{k} / 2}\left(\omega \sigma_{x}^{k} / 2\right) e^{i\left(\omega_{R F} t+\phi\right) \sigma_{z}^{k} / 2},
$$

where the transmitter's angular frequency $\omega_{R F}$, the initial phase $\phi$, and the power $\omega$ are tunable over an appropriate parameter range. NMR QIP has been extensively discussed in the literature [53]. At room temperature, NMR qubits exist in highly mixed, separable states and so NMR QIP relies on "pseudo-pure" (p.p.) states whose traceless (or "deviation") component is proportional to that of the corresponding pure state. The identity component of the density matrix is unobservable and, if the dynamics are unital, constant. The assumption of unital behavior has been validated experimentally, see Sect. IVD. Under these circumstances, the evolution of a p.p. state is equivalent to the one of the corresponding pure state. The 3-spin p.p. ground state $\varrho_{z}=|000\rangle\langle 000|$ was generated using standard gradient-pulse techniques whose details can be found in [54]. State preparation was experimentally verified by tomographically reconstructing the 3-spin density matrix [55]. A constant amount of identity component was added to all reconstructed density matrices such that the ground state fidelity with respect to the intended 3-spin p.p. state was optimized.

\section{B. Encoding and decoding quantum networks}

It is essential that the experimental procedure is designed to allow for the protection of an arbitrary one-qubit state, $\left|\psi_{\text {in }}\right\rangle=$ $\alpha|0\rangle+\beta|1\rangle$, with $\alpha$ and $\beta$ potentially unknown. Because collective errors affect the $Z$ subsystem hence induce a non-trivial evolution the encoded states, the decoding transformation $U_{d e c}$ must map the entire set of encoded basis states (17) back to the computational basis properly. Thus, a good decoding transformation provides an explicit realization of the mapping $U^{-1}$ appearing in 19]. Given $U_{d e c}$, an encoding transformation may be obtained by letting $U_{e n c}=U_{d e c}^{-1}$. Various choices are possible 
in principle, differing in the identification they establish between the state space of the abstract $L$ and $Z$ qubits and the physical qubits. The choice of $U_{d e c}$ we implemented maps $L, Z$ into the data qubit 2 and the ancilla qubit 3, respectively:

$$
\begin{aligned}
&|0\rangle_{L} \otimes|+1 / 2\rangle_{Z} \leftrightarrow\left|j=1 / 2, \ell=0, j_{z}=+1 / 2\right\rangle \mapsto|001\rangle \\
&|1\rangle_{L} \otimes|+1 / 2\rangle_{Z} \leftrightarrow\left|j=1 / 2, \ell=1, j_{z}=+1 / 2\right\rangle \mapsto|011\rangle \\
&|0\rangle_{L} \otimes|-1 / 2\rangle_{Z} \leftrightarrow\left|j=1 / 2, \ell=0, j_{z}=-1 / 2\right\rangle \mapsto|000\rangle \\
&|1\rangle_{L} \otimes|-1 / 2\rangle_{Z} \leftrightarrow\left|j=1 / 2, \ell=1, j_{z}=-1 / 2\right\rangle \mapsto|010\rangle .
\end{aligned}
$$

The fact that (31) only specifies $U_{d e c}$ on the $\mathcal{H}_{1 / 2}$ subspace is reflected by the fact that the value of the first ancilla qubit remains set to zero. $U_{d e c}$ is uniquely determined by also defining its action on the $\left|j=3 / 2, j_{z}\right\rangle$ states spanning $\mathcal{H}_{3 / 2}$ or, equivalently, the mapping with the remaining four computational basis states:

$$
\begin{array}{llr}
\left|j=3 / 2, j_{z}=+3 / 2\right\rangle & \mapsto & |100\rangle \\
\left|j=3 / 2, j_{z}=+1 / 2\right\rangle & \mapsto & -i|111\rangle \\
\left|j=3 / 2, j_{z}=-1 / 2\right\rangle & \mapsto & -i|110\rangle \\
\left|j=3 / 2, j_{z}=-3 / 2\right\rangle & \mapsto & |101\rangle .
\end{array}
$$

Having determined $U_{d e c}$, no general efficient procedure is known for designing a logical network of realizable one- and twoqubit operations effecting $U_{d e c}$. The implemented networks for $U_{d e c}$ and $U_{e n c}$ are shown in Fig. 2. Due to the weak strength of the $J_{13}$ coupling, no direct gates between spins 1 and 3 are used. For practical convenience, $U_{\text {enc }}$ is simplified by using qubit 3 as the information carrier (i.e., switching qubits 2 and 3 with respect to the output) and by taking advantage of the knowledge of the starting state $|00\rangle$ of the input ancillae. Thus, the implemented $U_{\text {enc }}$ effects the transformation

$$
U_{\text {enc }}\left|00 \psi_{\text {in }}\right\rangle \leftrightarrow\left(\alpha|0\rangle_{L} \otimes|-1 / 2\rangle_{Z}+\beta|1\rangle_{L} \otimes|-1 / 2\rangle_{Z}\right)=\left|\psi_{\text {in }}\right\rangle_{L} \otimes|-1 / 2\rangle_{Z} .
$$

The logical gates involved in the NS encoding and decoding circuits were first mapped into ideal pulse sequences via standard quantum network methods [53]. Pulses were then implemented by strongly modulating the internal Hamiltonian (29) of the alanine molecule with externally controlled RF magnetic fields as mentioned above. Details of pulse design can be found in [24]. Combinations of rotations that were used in multiple places in the sequence were merged into a single pulse and directly implemented. In practice, inaccurate preparation of the ancilla state $\left|a_{1} a_{2}\right\rangle=|00\rangle$ and operational errors may result in unintentionally populating states in the $\mathcal{H}_{3 / 2}$ subspace. Given the decoding action (32), this could contribute to observable signal upon discarding qubits 1 and 3 . The fact that contributions originating from $\mathcal{H}_{3 / 2}$ remained negligibly small was inferred in the implementation from the absence of appreciable double- and triple-coherence decay modes and from the stability of the observed signal against applied noise strength [30] (See also Sect. IVB).

It is worth emphasizing the difference between the ability to encode an arbitrary quantum state and the ability to accomplish a desired state preparation. While the latter is appropriate in the context of initializing a quantum algorithm, the former is crucial for quantum memory purposes (see also [39]) and for QEC in general. In the specific case, much simpler procedures would suffice for initializing the system into a desired, known NS state. For instance, one can observe that with an appropriate choice of basis a NS always contains a state which is the tensor product between a two-qubit singlet and a one-qubit logical state (see [2] for explicit encoded states alternative to (17)). Then, similar to the case of initialization in a DFS [56], synthesizing a NS state may be achieved by relying on a unitary transformation that prepares singlet states. Alternatively, one could exploit appropriate non-unitary control such as cooling, as suggested in [57]. For either DFSs or NSs, the existence of such initialization procedures does not automatically translate into the existence of an efficient way for effecting a general encoding. From this point of view, a systematic comparison of network complexity for DFSs vs NSs is worth being examined in more detail, and will be addressed elsewhere.

\section{Heisenberg representation of collective noise}

As discussed in Sect. IIA, it is unlikely that noise symmetries directly imply the preservation of one (or more) of the natural subsystem's DOF (such as the states of the physical spins). Yet, an abstract information-carrying subsystem not identifiable with any of the physical qubits exists through the subsystem identification (17) [2, 26]. The decoding operation corresponds to extracting the abstract protected DOF by mapping it to the natural DOF associated with the data qubit. In the abstract NS/syndrome representation, the desired error-correcting behavior translates into the property that collective errors act only on the $Z$ subsystem, as discussed earlier and explicitly verified in (20). An equivalent description can be constructed directly in terms of the physical DOFs by looking at the error algebra $\mathcal{A}_{c}$ in an appropriate Heisenberg representation determined by $U_{d e c}$.

Let $E_{a} \in \mathcal{A}_{c}$ be any collective error operator. The state of the entire system after it has been encoded into the NS, affected by the collective error, and then decoded from the NS is given by

$$
\left|\psi_{\text {out }}\right\rangle_{\mathrm{d}}|0\rangle_{\mathrm{a}_{1}}\left|\phi_{\text {out }}\right\rangle_{\mathrm{a}_{2}}=U_{\text {dec }} E_{a} U_{d e c}^{\dagger}\left|\psi_{\text {in }}\right\rangle_{\mathrm{d}}|0\rangle_{\mathrm{a}_{1}}\left|\phi_{\text {in }}\right\rangle_{\mathrm{a}_{2}}=E_{a}^{H}\left|\psi_{\text {in }}\right\rangle_{\mathrm{d}}|0\rangle_{\mathrm{a}_{1}}\left|\phi_{\text {in }}\right\rangle_{\mathrm{a}_{2}},
$$


where the Heisenberg-transformed error operator $E_{a}^{H}$ is defined by $E_{a}^{H}=U_{d e c} E_{a} U_{d e c}^{\dagger}$. Because $E_{a}$ is in $\mathcal{A}_{c}$, the action of $E_{a}^{H}$ can be inferred by knowing the transformed collective generators $J_{u}^{H}, u=x, y, z$. Using the decoding network of Fig. 2 2 , and recalling that $\mathrm{d}, \mathrm{a}_{1}, \mathrm{a}_{2}$ denote the data, the first and second ancilla qubit, respectively, one finds

$$
\begin{aligned}
& 2 J_{x}^{H}=E_{+}^{\mathrm{a}_{1}}\left(-\sigma_{x}^{\mathrm{a}_{2}}\right) \mathbb{1}^{\mathrm{d}}+E_{-}^{\mathrm{a}_{1}} \sigma_{x}^{\mathrm{a}_{2}}\left(\mathbb{1}^{\mathrm{d}}-2 \cos (\pi / 3) \sigma_{y}^{\mathrm{d}}-2 \sin (\pi / 3) \sigma_{z}^{\mathrm{d}}\right) \\
& 2 J_{y}^{H}=E_{+}^{\mathrm{a}_{1}}\left(-\sigma_{y}^{\mathrm{a}_{2}}\right) \mathbb{1}^{\mathrm{d}}+E_{-}^{\mathrm{a}_{1}} \sigma_{y}^{\mathrm{a}_{2}}\left(\mathbb{1}^{\mathrm{d}}-2 \cos (\pi / 3) \sigma_{y}^{\mathrm{d}}-2 \sin (\pi / 3) \sigma_{z}^{\mathrm{d}}\right) \\
& 2 J_{z}^{H}=E_{+}^{\mathrm{a}_{1}}\left(+\sigma_{z}^{\mathrm{a}_{2}}\right) \mathbb{1}^{\mathrm{d}}+E_{-}^{\mathrm{a}_{1}} \sigma_{z}^{\mathrm{a}_{2}}\left(\mathbb{1}^{\mathrm{d}}+2 \sigma_{z}^{\mathrm{d}}\right) .
\end{aligned}
$$

The above equations make it explicit that the action of any collective error is identity on the data bit, provided that the state of the first ancilla $a_{1}$ qubit is properly set to $|0\rangle$. This identity action is the counterpart of the one expressed in the Schrödinger picture by Eqs. (20). On the other hand, the second ancilla qubit $\mathrm{a}_{2}$ is in general evolved non-trivially by the noise. According to (35), the protection afforded by the NS can be equivatently understood as a rotation of the error algebra such that the abstract protected DOF is identified with the data qubit conditionally to the state of the first ancilla qubit.

\section{Design of the evolution period}

In order to verify the behavior of the NS code in a controlled setting, the delay period between encoding and decoding needs to be carefully designed so as to implement an effective evolution under a precisely known error model. An important building block for the procedure is the ability to implement a "no-op" evolution i.e., an evolution corresponding to the identity operation that provides the reference of no applied errors.

Additional constraints exist because, as mentioned, the assumptions made on $H_{S}$ in Sec. II are not achievable in the current experimental setting. In particular, $H_{S}$ does not itself respect the permutational symmetry of the collective error model, neither does it belong to the commutant $\tilde{\mathcal{A}}_{c}^{\prime}$ of the collective error algebra, causing the system to depart from the protected NS. The net, unwanted evolution induced by $H_{S}$ over the delay period can be averaged out by using refocusing techniques [41, 58]. The implemented sequences of $\pi$ pulses are depicted in Fig. 3 .

Because the system evolves through intermediate states outside the NS during the refocusing cycle, protection of the quantum data cannot be expected if noise is acting over the entire evolution period. However, what is crucial for verifying the robustness of the intended NS is that the information resides inside the protected space while the noise is applied. This can be achieved by making sure that the basic building block for engineering collective noise processes is a $z$-gradient, in which case $H_{S} \in \mathcal{A}_{z}^{\prime}$, and by applying the noise only during the sub-interval of the decoupling cycle corresponding to the identity frame (i.e., to the free evolution sub-interval) [17, 41, 59]. While this suffices for testing collective dephasing processes along $\hat{z}$, error algebrs of the form $\mathcal{A}_{x}, \mathcal{A}_{y}$ can also be probed, by simply sandwiching a $z$-noise process with the appropriate collective rotation pulse about either the $\hat{x}$ or $\hat{y}$ axis [44. Finally, cascades of noise blocks involving different axes were implemented by applying multiple noise blocks in series during a longer evolution period.

\section{E. Implementation of collective error models}

Two different engineered noise models were implemented to test both the weak and strong noise limits [30]. Both relied on linear magnetic field gradients in order to create an incoherent evolution over the spatial distribution of the sample [60]. The net phase evolution caused by a gradient pulse for a spin located $\delta_{z}$ from the center of the sample is given by

$$
\Delta \Phi\left(\delta_{z}\right)=\exp \left(-i \gamma \frac{d B_{z}}{d z} \delta_{z}\right)
$$

where $\gamma$ is the gyro-magnetic ratio of the nuclear species and determines how strongly the magnetic moment of the spin couples to the magnetic field. A strong gradient pulse causes spins at the edge of the sample to evolve through many cycles, producing an almost uniform distribution of phases across the sample. In the absence of molecular motion, the effects of this incoherent evolution could be refocused by a reverse gradient pulse. However, in liquid samples at room temperature, the spins are undergoing a random spatial diffusion that renders this incoherent process effectively irreversible, resulting in unrecoverable loss (i.e. decoherence) of the quantum information. The exponential attenuation of the signal associated with the combined gradient-diffusion process is described by a factor [60],

$$
A=\exp \left(-D \int k^{2}(t) d t\right)
$$

where $D$ is the molecular diffusion coefficient and $k(t)=\gamma t\left(d B_{z} / d z\right)$. For a gradient pulse of duration $\delta$, and a diffusion period of duration $\Delta$ followed by an inverse gradient also of duration $\delta$, the total attenuation of the coherence reduces to

$$
A=\exp \left(-D \gamma^{2}\left(d B_{z} / d z\right)^{2} \delta^{2}\left(\Delta+\frac{2 \delta}{3}\right)\right)
$$


Therefore, one can associate this evolution with a $T_{2}$-like process, $\exp (-t / \tau)$, where $1 / \tau$ represents the relevant, effective noise strength. By equating $A=\exp (-t / \tau)$, the elapsed time $t=\Delta+2 \delta$ being the duration of the gradient-diffusion process, the noise strength is given by

$$
\frac{1}{\tau}=D \gamma^{2}\left(d B_{z} / d z\right)^{2} \delta^{2} \frac{\Delta+\frac{2 \delta}{3}}{\Delta+2 \delta} .
$$

This reduces to the simpler expression quoted in [61] if $2 \delta \ll \Delta$. According to (39), the noise strength can be tuned by changing either the holding duration $\Delta$ or the gradient strength $d B_{z} / d z$. In order to keep the effects of other natural decoherence mechanisms fixed in different experimental runs, only the gradient strength was varied in practice (decoherent implementation). Each effective noise rate $1 / \tau$ was independently measured using a stimulated echo sequence [60].

If no reverse gradient is applied to refocus the magnetization, then the evolution remains incoherent until the ensemble signal is acquired, at which time the spatial degrees of freedom are traced over rendering the evolution effectively irreversible. Therefore, the crusher noise limit can be probed by using a strong gradient pulse with no reverse gradient pulse (incoherent implementation). The details of the no-op sequences and the different noise implementations are given in Fig. 3.

\section{RESULTS}

\section{A. Metric of control}

We used the entanglement fidelity [62] as the reliability measure quantifying how well quantum information was preserved under the evolution of an implemented super-operator $\mathcal{Q}$. Given an operator-sum decomposition of $\mathcal{Q}$ in terms of Kraus operators $\left\{A_{\mu}\right\}$, and an input state $\rho$, the entanglement fidelity can be computed as

$$
F_{e}(\mathcal{Q}, \rho)=\sum_{\mu} \operatorname{Tr}\left(\rho A_{\mu}\right) \operatorname{Tr}\left(\rho A_{\mu}^{\dagger}\right) .
$$

For a uniform distribution of input states, the maximally mixed input state, $\rho=\mathbb{1} / 2$, is used to characterize the un-biased channel performance. The entanglement fidelity can then be inferred from experimentally available data once it is expressed in terms of either pure state fidelities or relative input-output spin polarizations. Under the additional assumption of unital dynamics, i.e., $\mathcal{Q}(\mathbb{1})=\mathbb{1}$, the relevant expressions for single-qubit quantum process tomography are, respectively:

$$
F_{e}(\mathcal{Q})=\frac{1}{2}\left(F_{|+x\rangle}+F_{|+y\rangle}+F_{|+z\rangle}-1\right),
$$

or, equivalently,

$$
F_{e}(\mathcal{Q})=\frac{1}{4}\left(1+p_{x}+p_{y}+p_{z}\right) .
$$

In (41), the pure state input-output fidelity $F_{|\psi\rangle}=\operatorname{Tr}\{|\psi\rangle\langle\psi| \mathcal{Q}(|\psi\rangle\langle\psi|)\}$, and $|u\rangle, u=x, y, z$, are the eigenvectors of the corresponding Pauli operator with positive eigenvalue (thus, $|+z\rangle=|0\rangle,|-z\rangle=|1\rangle$ ) [30, 63]. In (42), $p_{u}=\operatorname{Tr}\left\{\sigma_{u} \mathcal{Q}\left(\sigma_{u}\right)\right\} / 2$ represents the relative output polarization given input $\sigma_{u}$ [40, 61, 63]. The implementation allowed to explicitly validate the consistency of the above expressions within experimental accuracy.

\section{B. Weak collective noise along a single axis}

The ability of the NS to protect quantum information against weak collective noise was tested using the gradient-diffusion techniques described above. The entanglement fidelity of the data qubit was experimentally determined from Eq. (41) as a function of noise strength for single axis collective noise. Separate experiments were carried over for extracting the behavior of both the NS-encoded qubit and the un-encoded data spin. The results are summarized in Fig. 4 . The measured curves are fitted to a decaying exponential function, $F_{e}=A \exp (-t / \tau)+B$, as expected for a dephasing channel induced by a single-axis noise mechanism. The un-encoded data's fit is characterized by $A=0.51 \pm 0.04$ and $B=0.43 \pm 0.03$, confirming the expected decaying contribution. The NS data's fit is instead characterized by $A=0.03 \pm 0.03$ for both $y$ and $z$ noise, with constant coefficients $B=0.62 \pm 0.02$ and $B=0.64 \pm 0.02$, respectively.

For all implementations, departures from the expected ideal behavior may be explained by pulse imperfections as well as by naturally occurring relaxation processes, whose effects are assumed to be independent of the applied noise strength. First, the fact that the decaying contribution (coefficient $A$ ) is small for both of the encoded situations indicates that the data resides in the 
NS during the application of the noise, which makes it insensitive to collective errors. Second, the fact that the constant term (coefficient $B$ ) is greater than 0.50 [64], confirms that quantum information is retained, in principle, for arbitrary noise strengths despite significant imperfections in the implementation of the encoding and decoding operations. Finally, the extrapolation of the data bounds the performance of the NS in the strong noise limit.

As discussed in Sect. IIG-H, unlike the case of a noiseless subspace the encoded NS states are not eigenstates of all noise operators. This implies, in general, a decay of the full-state fidelity due to evolution of the ancillae qubits under the action of the noise. Because the system always resides, within the experimental accuracy, in the spin-1/2 subspace, the ancilla $a_{1}$ remains unchanged by the application of the noise while the ancilla qubit $\mathrm{a}_{2}$, which is mapped from the syndrome $Z$, is decohered by the action of $y$ noise operators. For the case of collective $z$ noise, and with initialization in a fixed $j_{z}$ subspace, the system resides into a DFS, as noticed repeatedly. Thus, the state of the second ancilla qubit remains also unchanged under the $z$-dephasing process. These behaviors are experimentally confirmed in Fig. F. Note that because $\left|\mathrm{a}_{2}\right\rangle$ is always initialized to $|0\rangle, F_{e}$ does not provide an appropriate metric. An average input-output fidelity is evaluated instead, resulting from a uniform average over the data input states.

\section{Incoherent implementation to mimic strong collective noise}

A variety of incoherent collective noise processes were also implemented to explore the strong noise limit and to establish robustness of the implemented NS against the full, non-abelian $\mathcal{A}_{c}$. The entanglement fidelity data, again calculated via Eq. (41), are presented in Table [1] [30]. As expected, the un-encoded qubit's entanglement fidelity drops to 0.50 for single axis noise (corresponding to a full-strength dephasing channel), and to 0.25 for a cascade of non-commuting noise blocks (inducing a fullstrength depolarizing channel). For the NS-encoded case, the entanglement fidelity again departs from unity due to pulse errors and non-collective natural decoherence mechanisms, but it is changed only slightly under the action of different noise blocks. The significant improvement in the amount of information retained under a cascade of strong noise mechanisms confirms the expected benefits of the NS encoding already indicated by the weak noise data.

\section{Experimental determination of channel super-operators}

To gain further insight about the quantum processes realized in the experiment, the direct reconstruction of the super-operators describing some representative crusher dephasing channels was also obtained, leading to explicit sets of single-qubit Kraus operators. The measurement of the input-output relations for the set of input states $|+x\rangle\langle+x||+y\rangle,\langle+y||+z\rangle,\langle+z|$, and $\mid-$ $z\rangle\langle-z|$ is sufficient to allow the determination of a Kraus form for a desired super-operator [65, 66]. While this analysis assumes that the channel is trace-preserving, unitality is now not assumed, thereby allowing to independently test this assumption.

Table III collects the experimentally determined one-qubit super-operators and corresponding entanglement fidelities, calculated via Eq. (40) $(\rho=\mathbb{1} / 2)$ for five representative channels. Because the diagonal elements in each of the channel superoperators measure the relative polarizations $p_{u}$ introduced in $(42), F_{e}(\mathcal{Q})$ can equivalently be evaluated from the trace of the relevant super-operator representation (up to a factor 1/4).

First, the entanglement fidelity values are consistent with the ones calculated via Eq. (41). Second, the data show that the unitality assumption is broken by an average deviation no larger than $5 \%$. Finally, it is worth stressing that the measured superoperators contains complete information on the relative contributions of coherent vs decoherent errors. For instance, the purity of an output state $\rho_{\text {out }}$, given by $\xi=\operatorname{Tr}\left\{\rho_{\text {out }}^{2}\right\}$ for a given input state $\mathbb{1}, \sigma_{x}, \sigma_{y}, \sigma_{z}$ can be inferred from the sum of the squares of the elements of each row of the super-operator.

\section{CONCLUSIONS}

We presented a thorough theoretical and experimental investigation of the significance of a NS in the context of quantum information protection. This exploration demonstrates both the utility of the NS and its limitations. In particular, the assumption that the internal Hamiltonian is either proportional to one of the noise operators or that it is trivially zero is not valid for the NMR implementation, and is unlikely to be met exactly in any experimental realization of a quantum information processor. If such assumptions are at least approximately met, NSs represent the most efficient means for protecting quantum information in the presence of noise interactions with dominant symmetry components. For the example of the 3-qubit collective noise model, our experimental results convincingly demonstrate improvements in protecting information against a class of both abelian and nonabelian collective error models. Finally, experimentally reconstructed single-qubit super-operators provide detailed information about the properties and the relative contributions of coherent and decoherent errors in the implementation. 


\section{ACKNOWLEDGMENTS}

This work was supported by the National Security Agency and Advanced Research and Development Activity under Army Research Office contract number DAAD19-01-1-0519, by the Defense Sciences Office of the Defense Advanced Research Projects Agency under contract number MDA972-01-1-0003, and by the Department of Energy under contract W-7405-ENG36. L.V. also gratefully acknowledges support from a J. R. Oppenheimer Fellowship. We thank Grum Teklemariam and Nicolas Boulant for help with implementation, and Greg Boutis, Roberto Onofrio, and Seth Lloyd for valuable discussions.

[1] M. A. Nielsen and I. L. Chuang, Quantum Computation and Quantum Information (Cambridge University Press, Cambridge, UK, 2000).

[2] L. Viola, E. Knill, and R. Laflamme, J. Phys. A 34, 7067 (2001).

[3] P. W. Shor, in Proceedings of the 37th Symposium on the Foundations of Computer Science (IEEE Press, Los Alamitos, California, 1996).

[4] A.Yu.Kitaev, Russ. Math. Surv. 52, 1191 (1997).

[5] D. Aharonov and M. Ben-Or, in Proceedings of the 29th Annual ACM Symposium on the Theory of Computation (ACM Press, New York, 1996).

[6] E. Knill, R. Laflamme, and W. H. Zurek, Science 279, 342 (1998).

[7] J. Preskill, Proc. R. Soc. London A 454, 385 (1998).

[8] P. Zanardi and M. Rasetti, Phys. Rev. Lett. 79, 3306 (1997).

[9] L.-M. Duan and G.-C. Guo, Phys. Rev. Lett. 79, 1953 (1997).

[10] D. A. Lidar, I. L. Chuang, and K. B. Whaley, Phys. Rev. Lett. 81, 2594 (1998).

[11] E. Knill, R. Laflamme, and L. Viola, Phys. Rev. Lett. 84, 2525 (2000).

[12] L. Viola, E. Knill, and S. Lloyd, Phys. Rev. Lett. 85, 3520 (2000).

[13] S. De Filippo, Phys. Rev. A 62, 052307/1 (2000).

[14] P. Zanardi, Phys. Rev. A 63, 012301/1 (2001).

[15] L. Viola, E. Knill, and S. Lloyd, Phys. Rev. Lett. 82, 2417 (1999).

[16] P. Zanardi, Phys. Lett. A 258, 77 (1999).

[17] L. Viola, S. Lloyd, and E. Knill, Phys. Rev. Lett. 83, 4888 (1999).

[18] D. Vitali and P. Tombesi, Phys. Rev. A 59, 4178 (1999).

[19] L. Viola, Phys. Rev. A 66, 012307/1 (2002).

[20] L. Viola and E. Knill, quant-ph/0208056 (2002).

[21] R. Freeman, S. P. Kempsell, and M. H. Levitt, J. Magn. Res. 38, 453 (1980).

[22] A. J. Shaka and R. Freeman, J. Magn. Res. 55, 487 (1983).

[23] H. K. Cummins and J. A. Jones, New J. Phys. 2, 6.1 (2000).

[24] E. M. Fortunato, M. A. Pravia, N. Boulant, G. Teklemariam, T. F. Havel, and D. G. Cory, J. Chem. Phys. 116, 7599 (2002).

[25] E. Knill and R. Laflamme, Phys. Rev. A 55, 900 (1997).

[26] E. Knill, R. Laflamme, A. Ashikhmin, H. Barnum, L. Viola, and W. H. Zurek, quant-ph/0207170, to appear in Los Alamos Science 27 (2002).

[27] L.-A. Wu and D. A. Lidar, Phys. Rev. Lett. 88, 207902 (2002).

[28] D. Bacon, J. Kempe, D. A. Lidar, and K. B. Whaley, Phys. Rev. Lett. 85, 1758 (2000).

[29] P. Zanardi and S. Lloyd, quant-ph/0208132 (2002).

[30] L. Viola, E. M. Fortunato, M. A. Pravia, E. Knill, R. Laflamme, and D. G. Cory, Science 293, 2059 (2001).

[31] K. Kraus, States, Effects, and Operations (Springer-Verlag, New York, 1983).

[32] M. Burrow, Representation Theory of Finite Groups (Academic Press, New York, 1965).

[33] W. Arveson, An Invitation to $C^{*}$-Algebras (Springer-Verlag, New York, 1976), pp. 17-22.

[34] N. P. Landsman, math-ph/9807030 (1998).

[35] W. Thirring, A Course in Mathematical Physics (Springer-Verlag, New York, 1983), vol. 4, pp. 35-37.

[36] The "identity action" of the relevant error operators on a DOF of the system, as expressed by $\$$ ), remains the key (necessary and sufficient) condition for characterizing NSs without reference to a $\dagger$-closed algebraic structure as invoked here. Such generalization may be relevant to handle open-system evolutions directly described in terms of master equations, see for instance [37].

[37] J. Kempe, D. Bacon, D. A. Lidar, and K. B. Whaley, Phys. Rev. A 63, 042307 (2001).

[38] P. G. Kwiat, A. J. Berglund, J. B. Altepeter, and A. G. White, Science 290, 498 (2000).

[39] D. Kielpinski, V. Meyer, M. A. Rowe, C. A. Sackett, W. M. Itano, C. Monroe, and D. J. Wineland, Science 291, 1013 (2001).

[40] E. M. Fortunato, L. Viola, J. Hodges, G. Teklemariam, and D. G. Cory, New J. Phys. 4, 5.1 (2002).

[41] R. R. Ernst, G. Bodenhausen, and A. Wokaun, Principles of Nuclear Magnetic Resonance in One and Two Dimensions (Oxford University Press, Oxford, 1994).

[42] P. Zanardi, Phys. Rev. A 60, R729 (1999).

[43] J. Kempe, D. Bacon, D. A. Lidar, and K. B. Whaley, Phys. Rev. A 63, 042307 (2001).

[44] T. F. Havel, Y. Sharf, L. Viola, and D. G. Cory, Phys. Lett. A 280, 282 (2001).

[45] D. A. Lidar and L.-A. Wu, Phys. Rev. Lett. 88, 017905 (2001).

[46] L.-A. Wu, M. S. Byrd, and D. A. Lidar, Phys. Rev. Lett. 89, 127901 (2002). 
[47] A. G. Redfield (Academic Press, New York, 1965), vol. 1 of Advances in Magnetic Resonance.

[48] L. Viola and E. M. Fortunato, to appear in Los Alamos Science 27 (2002).

[49] A. Peres, Quantum Theory: Concepts and Methods (Kluwer Academic Publishers, Dordrecht, 1995), p. 132.

[50] For fixed $j_{z}$, each of the two-dimensional subspaces spanned by $\left\{\left|\ell, j_{z}\right\rangle, \ell=0,1\right\}$ is a one-qubit DFS under the collective dephasing error algebra $\mathcal{A}_{z}$.

[51] L. Masanes, G. Vidal, and J. I. Latorre, Quant. Inf. Comp. 2, 285 (2002).

[52] C.-P. Yang and J. Gea-Banacloche, Phys. Rev. A 63, 022311/1 (2001).

[53] D. G. Cory and et.al., Fortschr. Phys. 48, 875 (2000).

[54] G. Teklemariam, E. M. Fortunato, M. A. Pravia, T. F. Havel, and D. G. Cory, Phys. Rev. Lett. 86, 5845 (2001).

[55] I. L. Chuang, N. A. Gershenfeld, M. Kubinec, and D. W. Leung, Proc. R. Soc. London A 454, 447 (1998).

[56] P. Zanardi and F. Rossi, Phys. Rev. B 59, 8170 (1999).

[57] D. P. DiVincenzo, D. Bacon, J. Kempe, G. Burkard, and K. B. Whaley, Nature 408, 339 (2000).

[58] J. A. Jones and E. Knill, J. Magn. Res. 141, 322 (1999).

[59] Note that, if desired, application of the noise during the entire evolution period could be effected at the expenses of modulating the gradient Hamiltonian during each decoupling sub-interval, so as to compensate for the rotations due to the refocusing pulses. See [17] for related ideas on fast-switched control schemes.

[60] A. Sodickson and D. G. Cory, Progr. Nucl. Magn. Res. Spectrosc. 33, 77 (1998).

[61] D. G. Cory, W. Maas, M. Price, E. Knill, R. Laflamme, W. H. Zurek, T. F. Havel, and S. S. Somaroo, Phys. Rev.Lett. 81, 2152 (1998).

[62] B. Schumacher, Phys. Rev. A 54, 2614 (1996).

[63] E. Knill, R. Laflamme, R. Martinez, and C. Negrevergne, Phys. Rev. Lett. 86, 5811 (2001).

[64] C. Bennett, D. P. DiVincenzo, J. A. Smolin, and W. K. Wootters, Phys. Rev. A 54, 3824 (1996).

[65] A. M. Childs, I. L. Chuang, and D. W. Leung, Phys. Rev. A 64, 012314/1 (2001).

[66] T. F. Havel, quant-ph/0201127 (2002). 


\begin{tabular}{|cccccc|}
\hline Type of errors & Strength & Symmetries & $\operatorname{Dim}_{\mathcal{A}}$ & Error control code & Dim \\
\hline
\end{tabular}

TABLE I: Comparison between relevant error models on 3 qubits and corresponding error control strategies. The various columns list, in the order: the type of environment interaction (independent or collective); the relevant noise strength (or, equivalently, the order in time at which protection is sought [11], weak strength corresponding to first-order effects); the type of symmetry (permutation or axial); the dimension of an error basis for the appropriate error set; the most efficient error control code available for implementation on 3 qubits; the dimension of the state space $\mathcal{C}$ corresponding to the protected DOF. 3bit QEC means the standard QEC code for weak phase noise [1]. Note that finite-distance QEC could also be used to protect one qubit against general weak noise (either independent or collective), but the smallest such code requires 5 qubits [63].

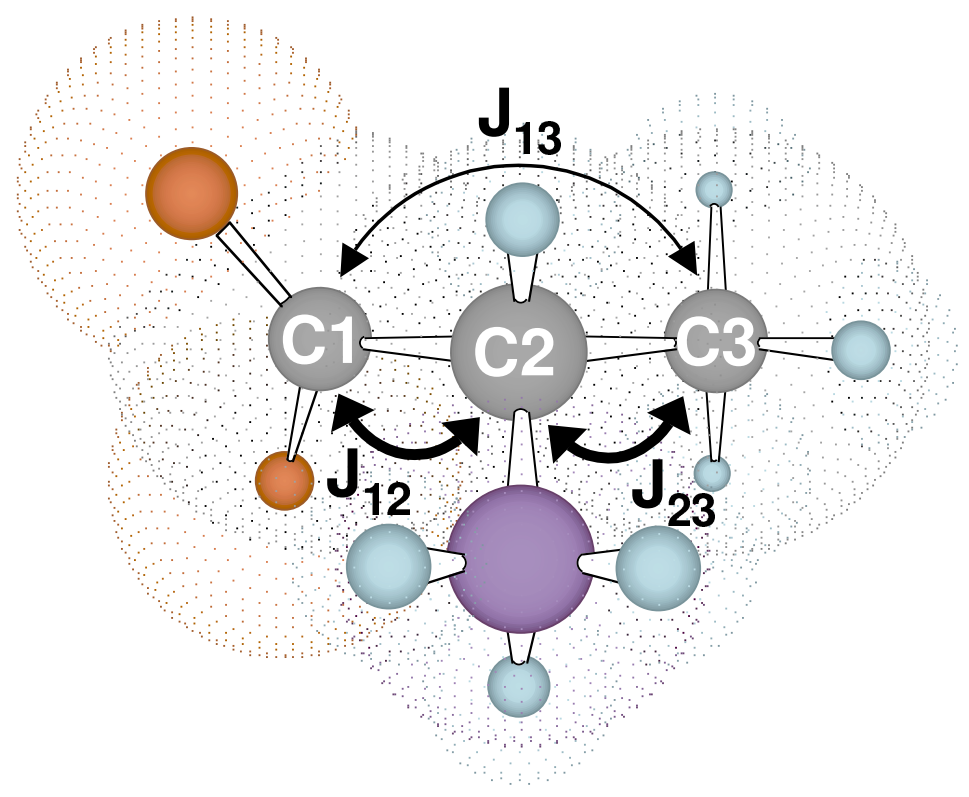

FIG. 1: Molecular structure of ${ }^{13} \mathrm{C}$ labeled alanine. The three carbon spins are used as qubits. In a reference frame that rotates at a frequency $75.4736434 \mathrm{MHz}$, the internal Hamiltonian is accurately described by $H_{S}=\pi\left[\nu_{1} \sigma_{z}^{(1)}+\nu_{2} \sigma_{z}^{(2)}+\nu_{3} \sigma_{z}^{(3)}+\left(J_{12} \sigma_{z}^{(1)} \sigma_{z}^{(2)}+J_{23} \sigma_{z}^{(2)} \sigma_{z}^{(3)}+\right.\right.$ $\left.\left.J_{13} \sigma_{z}^{(1)} \sigma_{z}^{(3)}\right) / 2\right)$, where $\nu_{1}-\nu_{0}=7167 \mathrm{~Hz}, \nu_{2}-\nu_{0}=-2286.5 \mathrm{~Hz}, \nu_{3}-\nu_{0}=-4881.4 \mathrm{~Hz}, J_{12}=54.1 \mathrm{~Hz}, J_{23}=35.0 \mathrm{~Hz}$, and $J_{13}=-1.3 \mathrm{~Hz}$. As it is impractical to directly utilize the coupling between qubits 1 and 3 due to its weak strength, conditional gates involving the pair of qubits $(1,3)$ were effectively replaced by sequences of operations involving pairs $(1,2)$ and $(2,3)$ in designing and executing quantum networks. The $T_{1}$ relaxation times for the three spins are approximately $21,2.5$, and $1.6 \mathrm{~s}$, while the $T_{2}$ times are about 550, 420, and $800 \mathrm{~ms}$, respectively. 


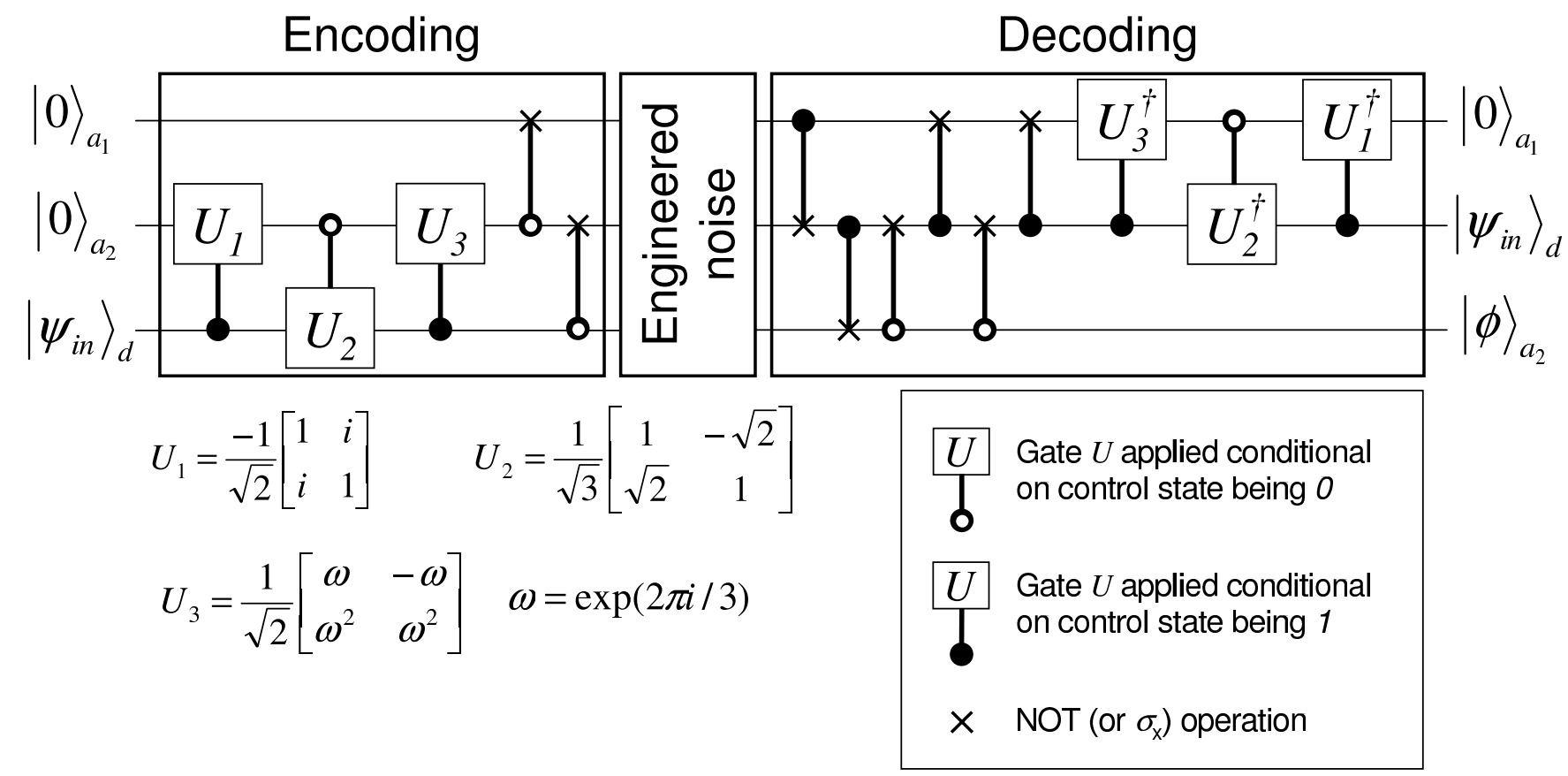

FIG. 2: Logical quantum network for the NS experiment. The information is initially stored in qubit 3, while the ancillae qubits 1 and 2 are initialized in the state $|0\rangle$. First, an encoding sequence $\left(U_{\text {enc }}\right)$ is applied in order to map the initial state of the system into the NS, according to (33). Next, the qubits are stored in memory while different noise processes are applied. Finally, the information is transferred to qubit 2 by the decoding sequence $\left(U_{d e c}\right)$. As noted in the text, $U_{e n c}$ is a simplified version of $U_{d e c}^{-1}$ obtained by exploiting the knowledge of the state of the ancillae qubits.

\begin{tabular}{|c|ccc|c|}
\hline Quantum process & $F_{|z\rangle}$ & $F_{|x\rangle}$ & $F_{|y\rangle}$ & $\mathbf{F}_{\mathbf{e}}$ \\
\hline \hline $\mathcal{Q}_{x, \text { un }}$ & 0.50 & 0.97 & 0.49 & $\mathbf{0 . 4 8}$ \\
\hline $\mathcal{Q}_{0, \mathrm{~ns}}$ & 0.84 & 0.74 & 0.78 & 0.68 \\
$\mathcal{Q}_{x, \text { ns }}$ & 0.79 & 0.74 & 0.78 & 0.66 \\
$\mathcal{Q}_{y, \text { ns }}$ & 0.81 & 0.77 & 0.82 & 0.70 \\
$\mathcal{Q}_{z, \text { ns }}$ & 0.86 & 0.72 & 0.76 & 0.67 \\
\hline $\mathcal{Q}_{z x, \text { un }}$ & 0.49 & 0.50 & 0.50 & $\mathbf{0 . 2 4}$ \\
\hline $\mathcal{Q}_{00, \text { ns }}$ & 0.80 & 0.79 & 0.80 & 0.70 \\
$\mathcal{Q}_{z x, \text { ns }}$ & 0.78 & 0.80 & 0.82 & 0.70 \\
$\mathcal{Q}_{z y, \text { ns }}$ & 0.79 & 0.80 & 0.82 & 0.70 \\
$\mathcal{Q}_{000, \text { ns }}$ & 0.77 & 0.79 & 0.78 & 0.67 \\
$\mathcal{Q}_{y z x, \text { ns }}$ & 0.75 & 0.80 & 0.77 & 0.66 \\
\hline \hline
\end{tabular}

TABLE II: Experimental data for implementation of various collective error models in the strong noise limit. The first column lists the one-bit quantum channels realized in the experiment. In addition to the applied error model, $\mathcal{E}_{x}, \mathcal{E}_{y}, \mathcal{E}_{z}, \mathcal{E}_{z x}, \mathcal{E}_{z y}, \mathcal{E}_{y z x}$, the channel label specifies whether (ns) or not (un) encoding and decoding procedures were implemented. The processes $\mathcal{Q}_{0, \text { ns }}, \mathcal{Q}_{00, \text { ns }}, \mathcal{Q}_{000, \text { ns }}$ differ in the duration over which the net identity evolution is applied. For each process, the input-output fidelities $F_{\left|\psi_{i n}\right\rangle}$ involved in the process tomography as well as the resulting entanglement fidelities $F_{e}$ are listed. Statistical uncertainties are $\sim 2 \%$, arising from errors in the tomographic density matrix reconstruction. 

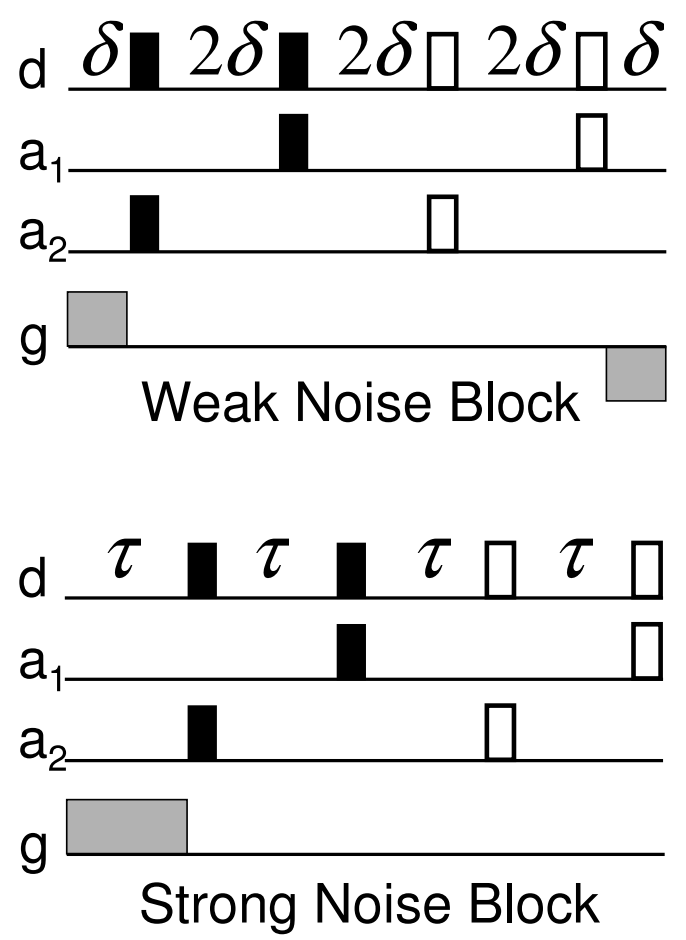

FIG. 3: No-op pulse sequences for both weak and strong noise applied to the data qubit $(\mathrm{d})$ and the two ancillae qubits $\left(\mathrm{a}_{1}\right.$ and $\left.\mathrm{a}_{2}\right)$. Black (white) boxes represent $\pi$ pulses about the $\hat{x}(-\hat{x})$ axis. The experimental delay times were $\delta \sim 5.5 \mathrm{~ms}$ and $\tau \sim 0.5 \mathrm{~ms}$. The bottom line (g) denotes the magnetic field gradient sequence used to introduce either weak (top) or strong (bottom) noise. As explained in Sect. IIID, all gradients were along the $\hat{z}$ axis and were only applied during the free evolution sub-interval of the whole evolution period. Collective noise along other axis was implemented by collective rotations sandwiching the entire noise block. Cascades of noise along non-commuting axes were implemented by applying multiple noise blocks in series. 


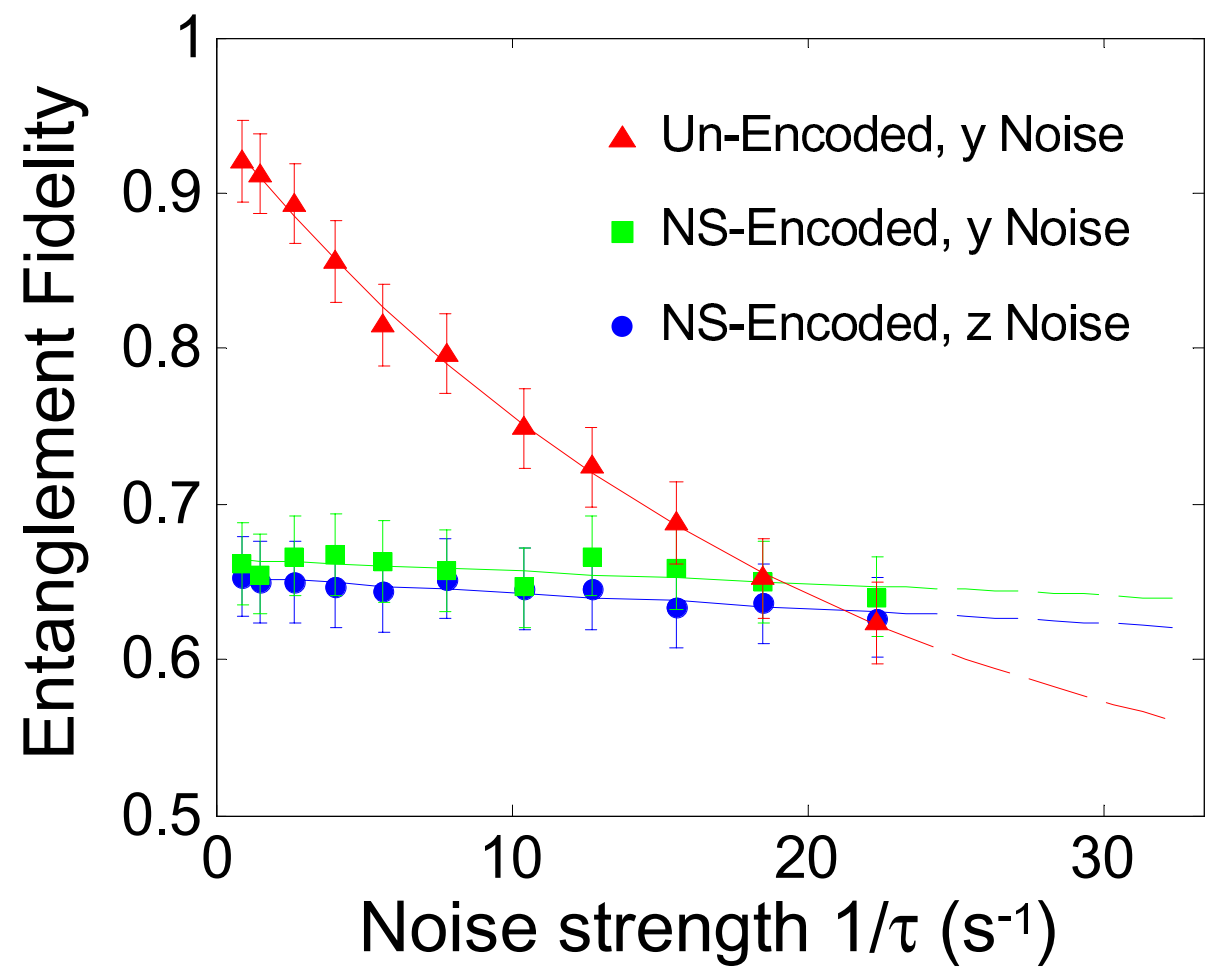

FIG. 4: Measured entanglement fidelities for single-axis collective noise in the weak noise regime along either the $\hat{y}$ axis (NS-encoded (squares) and un-encoded data (triangles)), or the $\hat{z}$ axis (NS-encoded data only (circles)). The decay of the un-encoded spin, $C_{3}$, was obtained by turning off the encoding and decoding sequences and by subjecting it to the noise sequence alone. The data are fit to an exponential decay function, with the interpolated (solid) and extrapolated (dashed) lines shown in the plot. 


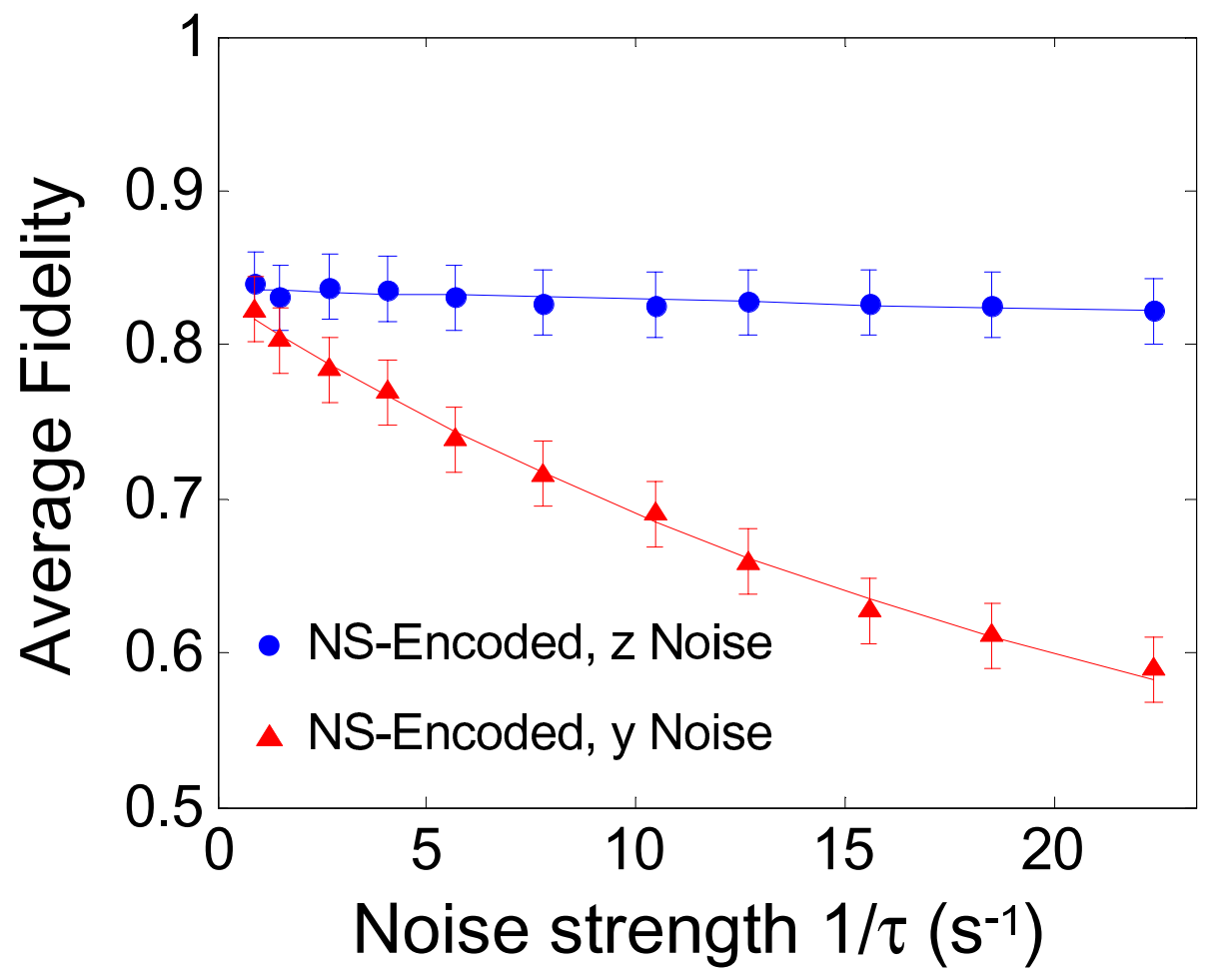

FIG. 5: Measured average state fidelity for the second ancilla qubit, $a_{2}$, for single-axis collective noise in the weak regime. Because the encoding transformation $U_{e n c}$ maps the initial state into an eigenstate of $J_{z}$ (see $\sqrt[33]{3}$ ), the ancilla qubit's state is unchanged by the application of noise processes along $\hat{z}$. This demonstrates that the encoding also maps the system into a one-qubit DFS against collective $z$-dephasing [50]. The case of collective $y$ noise demonstrates, however, that information is not stored in an eigenstate of a generic collective noise operator. 


$$
\begin{array}{rrrr}
\mathcal{Q}_{x, \text { un }} & \multicolumn{4}{c}{F_{e}=0.48} \\
\mathbb{1} & \sigma_{x} & \sigma_{y} & \sigma_{z} \\
\left(\begin{array}{rrrr}
1.00 & 0.01 & 0.00 & -0.01 \\
0.00 & 0.92 & 0.06 & -0.19 \\
0.00 & -0.02 & -0.02 & 0.02 \\
0.00 & 0.07 & 0.00 & 0.02
\end{array}\right) & \mathbb{1} \\
\sigma_{x} & \sigma_{y} \\
\sigma_{z}
\end{array}
$$

$$
\begin{array}{cccc}
\mathcal{Q}_{0, \mathrm{~ns}} & \multicolumn{3}{c}{F_{e}=0.69} \\
\mathbb{1} & \sigma_{x} & \sigma_{y} & \sigma_{z} \\
\left(\begin{array}{rrrr}
1.00 & -0.03 & 0.03 & 0.02 \\
0.00 & 0.53 & 0.29 & -0.16 \\
0.00 & -0.25 & 0.56 & 0.05 \\
0.00 & 0.10 & -0.07 & 0.67
\end{array}\right) & \mathbb{1} \\
\sigma_{x} & \sigma_{y} \\
\sigma_{z}
\end{array}
$$

$$
\begin{array}{rrrr}
\mathcal{Q}_{x, \mathrm{~ns}} & \multicolumn{4}{c}{F_{e}=0.67} \\
\mathbb{1} & \sigma_{x} & \sigma_{y} & \sigma_{z} \\
\left(\begin{array}{rrrr}
1.00 & -0.03 & 0.02 & 0.02 \\
0.00 & 0.54 & 0.28 & -0.21 \\
0.00 & -0.20 & 0.56 & 0.09 \\
0.00 & 0.21 & -0.07 & 0.58
\end{array}\right) & \mathbb{1} \\
\sigma_{x} & \sigma_{y} \\
\sigma_{z}
\end{array}
$$

\begin{tabular}{rrrr}
$\mathcal{Q}_{y, \text { ns }}$ & \multicolumn{5}{c}{$F_{e}=0.69$} \\
$\mathbb{1}$ & $\sigma_{x}$ & $\sigma_{y}$ & $\sigma_{z}$ \\
$\left(\begin{array}{rrrr}1.00 & 0.02 & 0.05 & 0.03 \\
0.00 & 0.55 & 0.27 & -0.16 \\
0.00 & -0.18 & 0.61 & 0.11 \\
0.00 & 0.16 & -0.09 & 0.60\end{array}\right)$ & $\mathbb{1}$ \\
$\sigma_{x}$ & $\sigma_{z}$
\end{tabular}

$$
\begin{aligned}
& \mathcal{Q}_{z, \mathrm{~ns}} \quad F_{e}=0.70 \\
& \begin{array}{llll}
11 & \sigma_{x} & \sigma_{y} & \sigma_{z}
\end{array} \\
& \left(\begin{array}{rrrr}
1.00 & -0.10 & 0.00 & 0.03 \\
0.00 & 0.56 & 0.29 & -0.10 \\
0.00 & -0.19 & 0.54 & 0.03 \\
0.00 & 0.12 & -0.07 & 0.67
\end{array}\right) \begin{array}{l}
\mathbb{1} \\
\sigma_{x} \\
\sigma_{y} \\
\sigma_{z}
\end{array}
\end{aligned}
$$

TABLE III: Experimentally reconstructed super-operators for the one-qubit channels. A fixed row represents the decomposition along the set of Pauli operators $\left\{\mathbb{1}, \sigma_{x}, \sigma_{y}, \sigma_{z}\right\}$ of the output corresponding to a given operator $\mathbb{1}, \sigma_{x}, \sigma_{y}$, or $\sigma_{z}$ taken as input. Because trace-preservation is assumed, the first entry in each row is constrained to unity, and the remaining entries in the first column to zero. Non-zero off-diagonal elements on the first row indicate the non-unitality of the channel. Entanglement fidelities, calculated via Eq. (40) are listed on top for each channel, and are also consistent with the values calculated from Eq. 42 , which equals $\operatorname{Tr}\{\mathcal{Q}\} / 4$. The above data may be interpreted as providing a matrix representation of the underlying super-operator as in [66] upon appropriately transposing rows and colums. 